5 July 2021

\title{
Oxygen-isotope effect on the in-plane penetration depth in cuprate superconductors
}

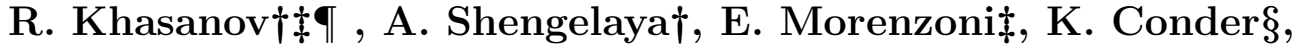 \\ I. M. Savić $\|$, and H. Keller $\dagger$ \\ $\dagger$ Physik-Institut der Universität Zürich, CH-8057 Zürich, Switzerland \\ $\ddagger$ Laboratory for Muon Spin Spectroscopy, Paul Scherrer Institut, CH-5232 Villigen \\ PSI, Switzerland \\ $\S$ Laboratory for Neutron Scattering, ETH Zürich and PSI Villigen, CH-5232 Villigen \\ PSI, Switzerland \\ || Faculty of Physics, University of Belgrade, 11001 Belgrade, Serbia and Montenegro
}

\begin{abstract}
Muon-spin rotation $(\mu \mathrm{SR})$ studies of the oxygen isotope $\left({ }^{16} \mathrm{O} /{ }^{18} \mathrm{O}\right)$ effect (OIE) on the in-plane magnetic field penetration depth $\lambda_{a b}$ in cuprate high-temperature superconductors (HTS) are presented. First, the doping dependence of the OIE on the transition temperature $T_{c}$ in various HTS is briefly discussed. It is observed that different cuprate families show a similar doping dependence of the OIE on $T_{c}$. Then, bulk $\mu \mathrm{SR}$, low-energy $\mu \mathrm{SR}$, and magnetization studies of the total and site-selective OIE on $\lambda_{a b}$ are described in some detail. A substantial OIE on $\lambda_{a b}$ was observed in various cuprate families at all doping levels, suggesting that cuprate HTS are nonadiabatic superconductors. The experiments clearly demonstrate that the total OIE on $T_{c}$ and $\lambda_{a b}$ arise from the oxygen sites within the superconducting $\mathrm{CuO}_{2}$ planes, demonstrating that the phonon modes involving the movement of planar oxygen are dominantly coupled to the supercarriers. Finally, it is shown that the OIE on $T_{c}$ and $\lambda_{a b}$ exhibit a relation that appears to be generic for different families of cuprate HTS. The observation of these unusual isotope effects implies that lattice effects play an essential role in cuprate HTS and have to be considered in any realistic model of high-temperature superconductivity.
\end{abstract}

PACS numbers: 76.75.+i, 74.72.-h, 82.20.Tr, 71.38

\footnotetext{
I e-mail: rustem.khasanov@psi.ch

Present address: Laboratory for Neutron Scattering, ETH Zürich and Paul Scherrer Institut, CH-5232 Villigen PSI, Switzerland; DPMC, Université de Genève, 24 Quai Ernest-Ansermet, 1211 Genève 4, Switzerland; Physik-Institut der Universität Zürich, Winterthurerstrasse 190, CH-8057 Zürich, Switzerland
} 


\section{Introduction}

Although the discovery of the cuprate high-temperature superconductors (HTS) [1] in 1986 triggered world-wide an enormous effort to understand these novel materials, there is at present still no convincing microscopic theory describing the mechanism of superconductivity. Due to the high values of the superconducting transition temperature $T_{c}$ and the early observation of a tiny oxygen-isotope effect in optimally doped $\mathrm{YBa}_{2} \mathrm{Cu}_{3} \mathrm{O}_{7-\delta}$ [2, 3, 4], many theoreticians came to the conclusion that the electronphonon interaction cannot be responsible for high-temperature superconductivity. As a result, alternative pairing mechanisms of purely electronic origin were proposed. However, the assumption that $T_{c}$ cannot be higher than $30 \mathrm{~K}$ within a phonon-mediated pairing mechanism is not justified [5. A prominent example is the recent discovery of superconductivity in $\mathrm{MgB}_{2}\left[6\right.$, with a $T_{c} \approx 39 \mathrm{~K}$ which is accepted to be a purely phononmediated superconductor. There is increasing experimental evidence from recent work, such as neutron scattering [7, 8, angle resolved photoemission spectroscopy [9, 10], and isotope effect studies (this work) that lattice effects play an essential role in the basic physics of HTS and have to be considered in reliable theoretical models [11, 12.

It is well known that the observation of an isotope effect on $T_{c}$ in conventional superconductors was crucial in the development of the microscopic BCS theory. The isotope shift may be quantified in terms of the relation

$$
T_{c} \propto M^{-\alpha}, \quad \alpha=-d \ln T_{c} / d \ln M,
$$

where $M$ is the isotope mass, and $\alpha$ is the isotope-effect exponent. In the simplest case of weak-coupling BCS theory $T_{c} \propto M^{-1 / 2}$ and $\alpha_{\mathrm{BCS}} \simeq 0.5$, in agreement with a number of experiments on conventional metal superconductors. However, there are exceptions of this rule such as $\mathrm{Zr}$ and $\mathrm{Ru}$ for which $\alpha \simeq 0$.

The conventional phonon-mediated theory is based on the Migdal adiabatic approximation in which the density of states at the Fermi level $N(0)$, the electronphonon coupling constant $\lambda_{e p}$, and the effective supercarrier mass $m^{*}$ are all independent of the mass $M$ of the lattice atoms. However, if the interaction between the carriers and the lattice ions is strong enough, the Migdal approximation is no more valid 13. Therefore, in contrast to ordinary metals, unconventional isotope effects on various quantities, such as the superconducting transition temperature and the magnetic penetration depth are expected for a non-adiabatic superconductor.

In 1990 the University of Zurich group started a project on isotope effect on cuprates that was initiated by K. Alex Müller. Here we briefly review some of our results. They include unconventional oxygen isotope $\left({ }^{16} \mathrm{O} /{ }^{18} \mathrm{O}\right)$ effects $(\mathrm{OIE})$ in HTS on the transition temperature and the in-plane magnetic penetration depth. For a detailed description of our work we refer to Refs. [14, 15, 16, 17, 18, 19, 20, 21, 22. In this review it is demonstrated that the muon spin rotation $(\mu \mathrm{SR})$ technique is a very powerful and unique tool to investigate the OIE on the magnetic penetration depth in HTS. In particular, the novel low-energy $\mu \mathrm{SR}$ technique (LE $\mu \mathrm{SR}$ ) 23] allows a direct and very 
accurate measurement of the magnetic penetration depth in HTS and with that a reliable measurement of the OIE on $\lambda$.

The paper is organized as follows. In Sec. 2 we describe the sample preparation and the oxygen exchange procedure. Some results of the OIE on the transition temperature $T_{c}$ obtained for different cuprate families are presented in Sec. 3. Sec. 4 comprises studies of the OIE on the in-plane penetration depth $\lambda_{a b}$ in $\mathrm{Y}_{1-x} \operatorname{Pr}_{x} \mathrm{Ba}_{2} \mathrm{Cu}_{3} \mathrm{O}_{7-\delta}$ and $\mathrm{La}_{2-x} \mathrm{Sr}_{x} \mathrm{CuO}_{4}$ by means of bulk $\mu \mathrm{SR}$ and in optimally doped $\mathrm{YBa}_{2} \mathrm{Cu}_{3} \mathrm{O}_{7-\delta}$ by means of LE $\mu \mathrm{SR}$. Furthermore, results of the site-selective OIE on $\lambda_{a b}$ in $\mathrm{Y}_{0.6} \mathrm{Pr}_{0.4} \mathrm{Ba}_{2} \mathrm{Cu}_{3} \mathrm{O}_{7-\delta}$ obtained by bulk $\mu \mathrm{SR}$ are reported. In Sec. 5 we discuss implications of the OIE on $\lambda_{a b}$ and the empirical relation between the isotope effect on $T_{c}$ and $\lambda_{a b}$ observed for different HTS families. The conclusions follow in Sec. 6.

\section{Sample preparation and oxygen isotope exchange}

For isotope effect studies it is important to have isotope substituted samples of the same quality. In the case of ${ }^{16} \mathrm{O} /{ }^{18} \mathrm{O}$ oxygen substituted samples it means that both samples should have (i) exactly the same oxygen stoichiometry, (ii) the same oxygen distribution within the sample, and (iii) the same grain size distribution (in the case of powder samples). The schematic view of the experimental setup used for the oxygen

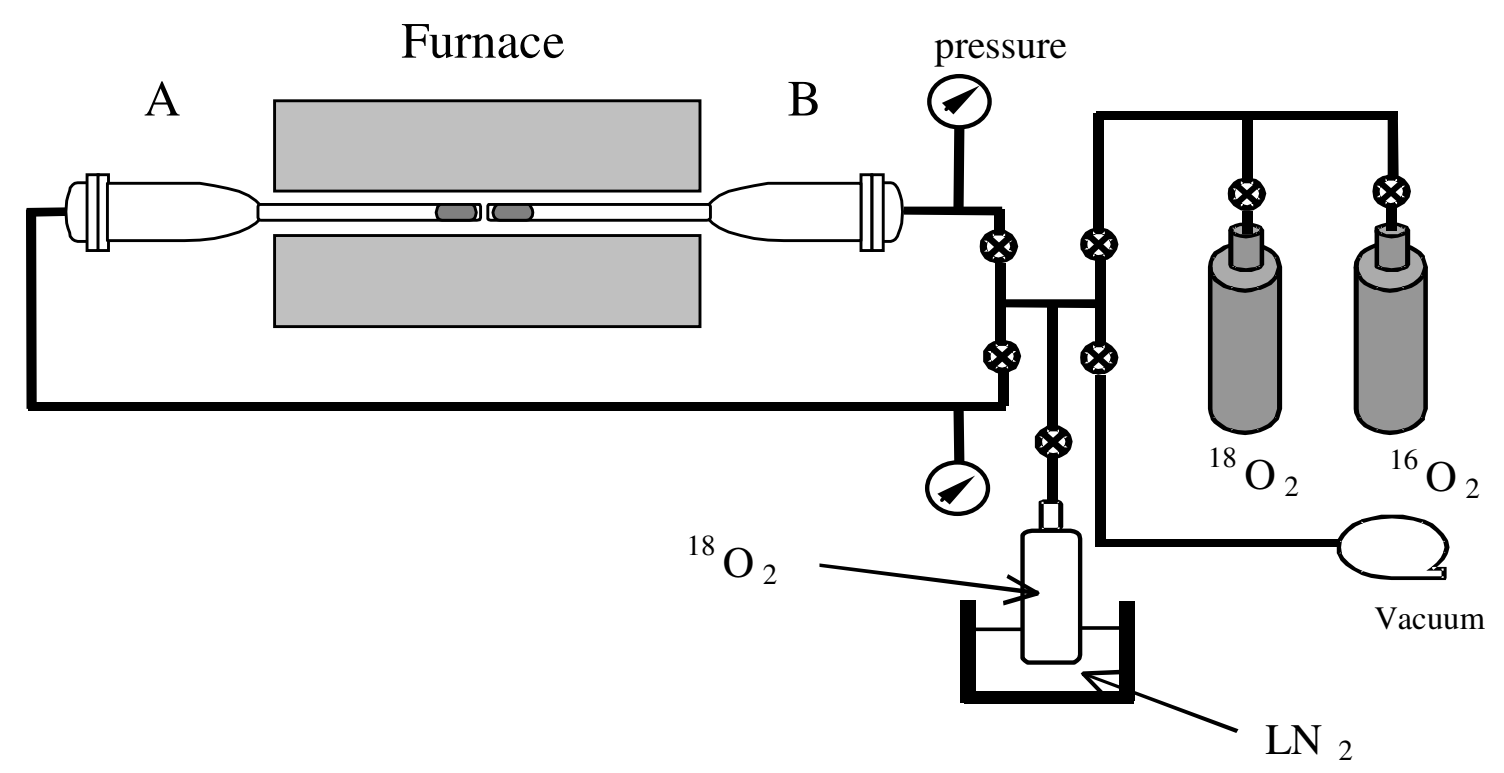

Figure 1. Experimental setup for preparation of the ${ }^{16} \mathrm{O} /{ }^{18} \mathrm{O}$ substituted samples.

isotope exchange is shown in Fig. 1 24]. In order to ensure that the substituted samples are subject to the same thermal history, the annealing (in ${ }^{16} \mathrm{O}_{2}$ and ${ }^{18} \mathrm{O}_{2}$ ) is performed simultaneously. In chamber A the isotope exchange $\left({ }^{18} \mathrm{O}\right)$ and in chamber $\mathrm{B}$ an identical 
process in normal oxygen $\left({ }^{16} \mathrm{O}\right)$ takes place. A liquid nitrogen trap is used to condense (and recycle) the expensive ${ }^{18} \mathrm{O}_{2}$ after the exchange process is finished. The exchange apparatus is equipped with a mass spectrometer (not shown), which allows to view the progress of the isotope exchange.

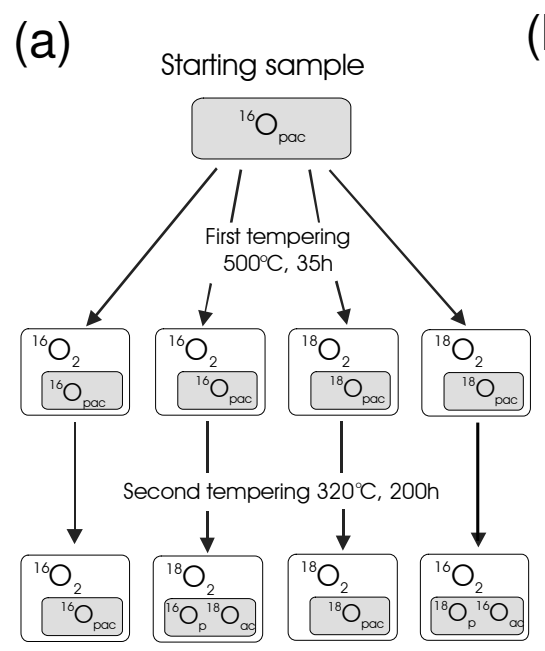

(b)

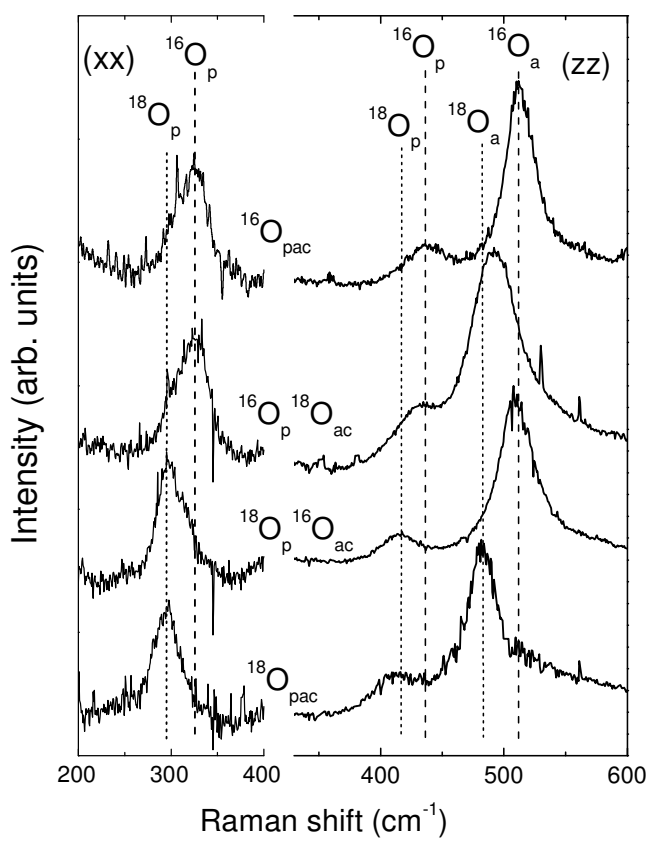

Figure 2. (a) Schematic diagram showing the oxygen isotope $\left({ }^{16} \mathrm{O} /{ }^{18} \mathrm{O}\right)$ substitution procedure to prepare completely and site-selective substituted samples. The first step is used to prepare completely oxygen substituted samples. The second step is used to prepare the site-selective samples from the completely substituted ones. (b) Roomtemperature Raman spectra of the completely and site-selective oxygen substituted $\mathrm{Y}_{0.6} \mathrm{Pr}_{0.4} \mathrm{Ba}_{2} \mathrm{Cu}_{3} \mathrm{O}_{7-\delta}$ samples. For $x x$ polarization the line corresponds to the outof-phase motion of the planar oxygen atoms $\left({ }^{16} \mathrm{O}, 325 \mathrm{~cm}^{-1} ;{ }^{18} \mathrm{O}, 294 \mathrm{~cm}^{-1}\right)$. For $z z$ polarization the two lines correspond to the in-phase motion of the $\mathrm{CuO}_{2}$ plane oxygen $\left({ }^{16} \mathrm{O}, 436 \mathrm{~cm}^{-1} ;{ }^{18} \mathrm{O}, 415 \mathrm{~cm}^{-1}\right)$ and to the bond-stretching mode of the apical oxygen $\left({ }^{16} \mathrm{O}, 512 \mathrm{~cm}^{-1} ;{ }^{18} \mathrm{O}, 482 \mathrm{~cm}^{-1}\right)$. After [21].

The procedure to prepare completely oxygen substituted and site-selective oxygen substituted $\mathrm{Y}_{1-x} \mathrm{Pr}_{x} \mathrm{Ba}_{2} \mathrm{Cu}_{3} \mathrm{O}_{7-\delta}$ samples (which are mostly described in this review) is schematically shown in Fig. 2(a). In the first step $\left[500^{\circ} \mathrm{C}, 35 \mathrm{~h}\right.$ at 1.2 bar in ${ }^{16} \mathrm{O}_{2}\left({ }^{18} \mathrm{O}_{2}\right)$ gas] completely oxygen substituted samples $\left({ }^{16} \mathrm{O}_{\text {pac }}\right.$ and $\left.{ }^{18} \mathrm{O}_{\text {pac }}\right)$ are prepared. Here indexes $p, a$, and $c$ stay for the planar (within $\mathrm{CuO}_{2}$ planes), the apical and the chain oxygen, respectively. In order to prepare site-selective substituted samples after the first step they were grouped in two pairs. Then two site-selective samples $\left({ }^{16} \mathrm{O}_{\mathrm{p}}{ }^{18} \mathrm{O}_{\mathrm{ac}}\right.$ and $\left.{ }^{18} \mathrm{O}_{\mathrm{p}}{ }^{16} \mathrm{O}_{\mathrm{ac}}\right)$ were prepared via annealing one ${ }^{16} \mathrm{O}_{\mathrm{pac}}$ sample in a ${ }^{18} \mathrm{O}_{2}$ atmosphere and one 
${ }^{18} \mathrm{O}_{\text {pac }}$ sample in ${ }^{16} \mathrm{O}_{2}$ gas $\left(330^{\circ} \mathrm{C}, 150 \mathrm{~h}, 1.2 \mathrm{bar}\right)$ [see Fig. 2(a)]. The other two samples (one ${ }^{16} \mathrm{O}_{\text {pac }}$ and one ${ }^{18} \mathrm{O}_{\text {pac }}$ ) were simultaneously annealed in the same atmosphere as before in order to have the reference samples following the same thermal history.

The results of the oxygen exchange can be checked by Raman spectroscopy. Fig. 2(b) shows Raman spectra with $z z$ and $x x$ polarizations of completely and siteselective oxygen substituted $\mathrm{Y}_{0.6} \mathrm{Pr}_{0.4} \mathrm{Ba}_{2} \mathrm{Cu}_{3} \mathrm{O}_{7-\delta}$ samples [21]. In the ${ }^{18} \mathrm{O}_{\text {pac }}$ sample, the Raman lines are all shifted to lower frequencies in agreement with the results of Zech et al. [25, indicating a nearly complete exchange of ${ }^{16} \mathrm{O}$ with ${ }^{18} \mathrm{O}$. In the siteselective sample ${ }^{16} \mathrm{O}_{\mathrm{p}}{ }^{18} \mathrm{O}_{\mathrm{ac}}$, only the position of the apical oxygen line is shifted to lower frequency, whereas the lines corresponding to the plane oxygen stay the same [apart from a small shift of one Raman line $\left(433 \mathrm{~cm}^{-1}\right.$ instead of $\left.436 \mathrm{~cm}^{-1}\right)$, probably due to a small unintentional partial substitution by $\left.{ }^{18} \mathrm{O}\right]$. Note that the apical line $\left(492 \mathrm{~cm}^{-1}\right)$ is also slightly shifted from the expected $482 \mathrm{~cm}^{-1}$, indicating that the oxygen exchange for the apical and chain oxygen is slightly uncomplete. In the ${ }^{18} \mathrm{O}_{\mathrm{p}}{ }^{16} \mathrm{O}_{\mathrm{ac}}$ sample only the two planar lines are shifted, while the apical line stays the same.

\section{Oxygen isotope effect on the transition temperature $T_{c}$}
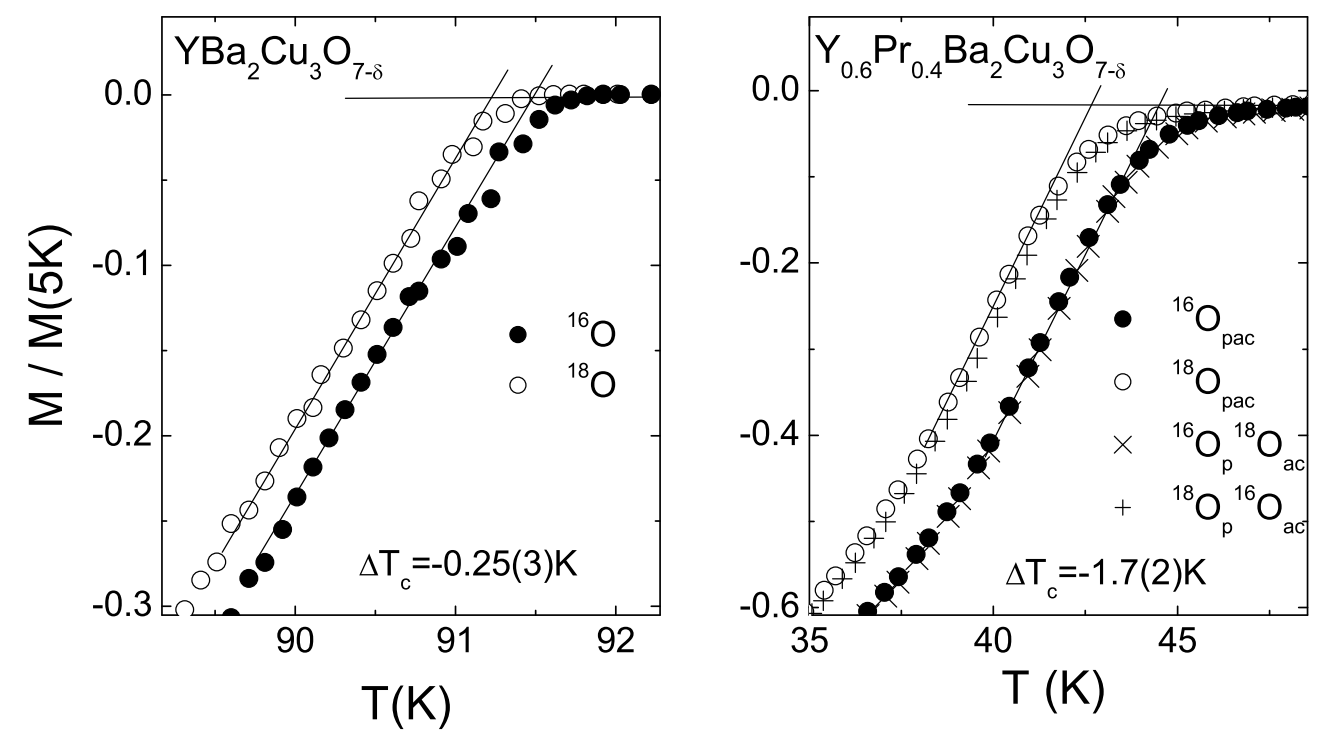

Figure 3. Section near $T_{c}$ of the normalized (to the value at $5 \mathrm{~K}$ ) magnetization curves $(1 \mathrm{mT}, \mathrm{FC})$ of the completely oxygen substituted $\mathrm{YBa}_{2} \mathrm{C}_{3} \mathrm{O}_{7-\delta}$ (a) and the site-selective oxygen substituted $\mathrm{Y}_{0.6} \mathrm{Pr}_{0.4} \mathrm{Ba}_{2} \mathrm{Cu}_{3} \mathrm{O}_{7-\delta}$ samples (b). After [21].

The importance of phonons for the pairing mechanism of conventional superconductors (including doped fullerenes [26] and $\mathrm{MgB}_{2}$ [27]) was provided by 
measurements of the isotope effect (IE) on $T_{c}$. The BCS theory predicts that

$$
k_{B} T_{c}=1.13 \hbar \omega \exp \left(-\frac{1}{N(0) V}\right),
$$

where $\omega$ is a typical phonon frequency (e.g. the Debye frequency $\omega_{D}$ ). The electron phonon coupling is given by the product of the electron-phonon interaction constant $V$ and the electronic density of states at the Fermi surface $N(0)$, both of which are assumed to be independent of the ion mass $M$. Eq. (22) implies an isotope-mass dependence of $T_{c}\left(T_{c} \propto \omega \propto 1 / \sqrt{M}\right)$, characterized by the isotope effect exponent $\alpha=1 / 2$. This is in agreement with isotope effect results reported for many nontransition metal superconductors (e.g. $\mathrm{Hg}$, Sn and Pd). However, as mentioned above, there are exceptions as $\mathrm{Zr}$ and $\mathrm{Ru}$ with $\alpha \simeq 0$.

Since 1987 a number of oxygen isotope effect investigations on $T_{c}$ were performed in most families of HTS. The first OIE experiments were done on optimally doped samples, showing no significant isotope shift [2, 4]. However later experiments revealed a small but finite dependence of $T_{c}$ on the oxygen isotope mass $M_{\mathrm{O}}$ [3, 28, 29. It is now well established that the OIE on $T_{c}$ is doping dependent [29, 30]. As an example, Fig. 3 shows the temperature dependence of the magnetization in the vicinity of $T_{c}$ in optimally doped ${ }^{16} \mathrm{O} /{ }^{18} \mathrm{O} \mathrm{YBa}_{2} \mathrm{Cu}_{3} \mathrm{O}_{7-\delta}$ samples and site-selective oxygen exchanged $\mathrm{Y}_{0.6} \mathrm{Pr}_{0.4} \mathrm{Ba}_{2} \mathrm{Cu}_{3} \mathrm{O}_{7-\delta}$ samples. It is seen that the isotope shift on $T_{c}$ increases with decreasing doping (increasing $\operatorname{Pr}$ concentration) quite substantially: from $\Delta T_{c}=-$ $0.25(3) \mathrm{K}$ in optimally doped $\mathrm{YBa}_{2} \mathrm{Cu}_{3} \mathrm{O}_{7-\delta}$ to $\Delta T_{c}=-1.7(2) \mathrm{K}$ in highly underdoped $\mathrm{Y}_{0.6} \mathrm{Pr}_{0.4} \mathrm{Ba}_{2} \mathrm{Cu}_{3} \mathrm{O}_{7-\delta}$.

The oxygen-isotope exponent $\alpha_{O}=-\mathrm{d} \ln T_{c} / \mathrm{d} \ln M_{\mathrm{O}}$ as a function of doping shows a trend that appears to be generic for all families of cuprate superconductors [15, 16, 18, 28, 31]. In the underdoped region $\alpha_{O}$ is large (even exceeding the BCS value $\alpha=1 / 2$ ) and becomes small in the optimally doped and overdoped regime (see Fig. (4). Moreover, the copper-isotope $\left({ }^{63} \mathrm{Cu} /{ }^{65} \mathrm{Cu}\right)$ exponent $\alpha_{\mathrm{Cu}}$ shows a similar trend as $\alpha_{\mathrm{O}}$ [34, 35, 36, 37]. Both exponents increase monotonically with decreasing doping level (or $T_{c}$ ). For $\mathrm{Y}_{1-x} \mathrm{Pr}_{x} \mathrm{Ba}_{2} \mathrm{Cu}_{3} \mathrm{O}_{7-\delta}$ and $\mathrm{Y}_{1-x} \mathrm{Pr}_{x} \mathrm{Ba}_{2} \mathrm{Cu}_{4} \mathrm{O}_{8}$ close to optimal doping $\alpha_{\mathrm{Cu}} \simeq \alpha_{\mathrm{O}}$, whereas in the deeply underdoped regime $\alpha_{\mathrm{Cu}} \simeq 0.7 \alpha_{\mathrm{O}}$ [18, 35, 36, 37.

The observation of OIE on $T_{c}$ indicates that lattice vibrations in the $\mathrm{CuO}_{2}$ planes are relevant for the occurrence of superconductivity. From this point of view, one would expect that the planar oxygen modes give rise to larger OIE on $T_{c}$ than the apical and/or chain oxygen modes. The first reliable site-selective OIE (SOIE) studies of $T_{c}$ were performed by Zech et al. [25]. It was shown that in optimally doped $\mathrm{YBa}_{2} \mathrm{Cu}_{3} \mathrm{O}_{7-\delta}$ the planar oxygen mainly $(\geq 80 \%)$ contribute to the total OIE on $T_{c}$. These results were confirmed by Zhao et al. [31] and later by Khasanov et al. [21]. Both groups [31, 21] found that in underdoped $\mathrm{Y}_{1-x} \mathrm{Pr}_{x} \mathrm{Ba}_{2} \mathrm{Cu}_{3} \mathrm{O}_{7-\delta}$ the predominant contribution (100\% within error bar) arises from the oxygen within the superconducting $\mathrm{CuO}_{2}$ planes [see Fig. [3(b)]. 


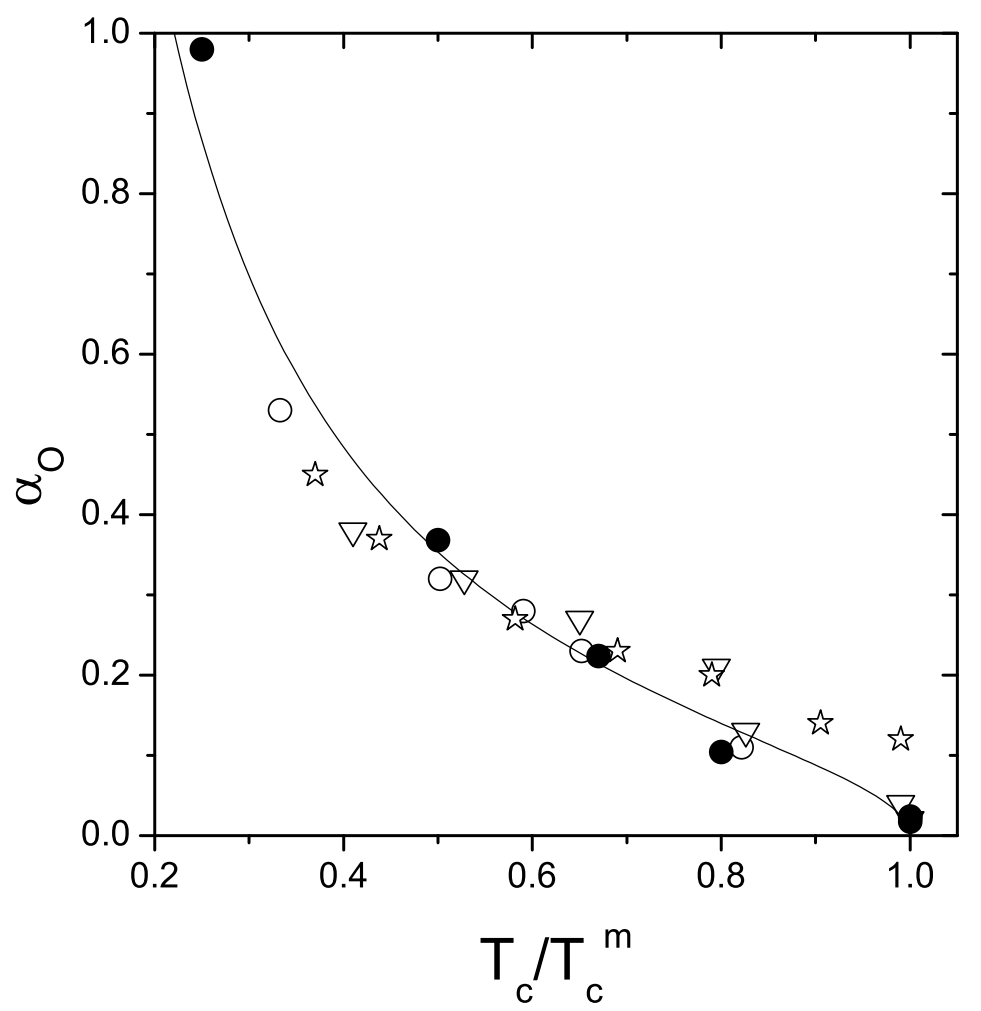

Figure 4. Oxygen isotope effect exponent $\alpha_{\mathrm{O}}$ versus reduced temperature $\bar{T}_{c}=$ $T_{c} / T_{c}^{m}$ ( $T_{c}^{m}$ is the maximum transition temperature of a given family of HTS). The open circles are $\mathrm{Y}_{1-x} \mathrm{Pr}_{x} \mathrm{Ba}_{2} \mathrm{Cu}_{3} \mathrm{O}_{7-\delta}$ data from 28. Open down triangles are data of $\mathrm{YBa}_{2-x} \mathrm{La}_{x} \mathrm{Cu}_{3} \mathrm{O}_{7}$ taken from 32]. Open stars are data of $\mathrm{La}_{1.85} \mathrm{Sr}_{0.15} \mathrm{Cu}_{1-x} \mathrm{Ni}_{x} \mathrm{O}_{4}$ from [33. Closed circles correspond to $\mathrm{Y}_{1-x} \mathrm{Pr}_{x} \mathrm{Ba}_{2} \mathrm{Cu}_{3} \mathrm{O}_{7-\delta}$ data from [20, 22] and unpublished data (this work). The solid line corresponds to $\alpha_{\mathrm{O}}=0.25 \sqrt{\left(1-\bar{T}_{c}\right)} / \bar{T}_{c}$ ) from [30].

\section{Oxygen isotope effect on the in-plane magnetic penetration depth $\lambda_{a b}$}

The conventional phonon-mediated theory of superconductivity (standard BCS theory) is based on the Migdal adiabatic approximation in which the effective supercarrier mass $m^{*}$ is independent of the mass $M$ of the lattice atoms. However, if the interaction between the carriers and the lattice is strong enough, the Migdal adiabatic approximation breaks down and $m^{*}$ depends on $M$ (see, e.g. [13]). A significant experiment to explore a possible coupling of the supercarriers to the lattice is an isotope effect study of the magnetic field penetration depth $\lambda$.

For a general Fermi surface the zero-temperature magnetic penetration depth $\lambda_{i i}(0)$ may be written as an integral over the Fermi surface [38]:

$$
\frac{1}{\lambda_{i i}(0)^{2}}=\frac{\mu_{0}}{2 \pi^{2} h} \oint d S_{F} \frac{v_{F_{i}} v_{F_{i}}}{\left|v_{F}\right|},
$$


where $i$ denotes the crystallographic axes $(a, b, c)$ and $v_{F_{i}}$ is the Fermi velocity. For the special cases of spherical or ellipsoidal Fermi surfaces, Eq. 3 leads to the London expression

$$
\frac{1}{\lambda_{i i}(0)^{2}}=\mu_{0} e^{2} \frac{n_{s}}{m_{i i}^{*}}
$$

where $n_{s}$ is the superconducting carrier density and $m_{i i}^{*}$ is the effective mass of the carriers. For a general Fermi surface, it is convenient to parameterize experimental data by means of Eq. 4. It should be noted, however, that in this case $m_{i i}^{*}$ is not directly related the band mass, except for spherical or ellipsoidal Fermi surfaces [38]. For HTS, which are superconductors in the clean limit, we may parameterize the in-plane penetration depth $\lambda_{a b}$ in terms of the relation:

$$
1 / \lambda_{a b}^{2}(0) \sim n_{s} / m_{a b}^{*},
$$

where $m_{a b}^{*}$ is the in-plane effective mass (not band mass) of the charge carriers. According to Eq. 5. this implies that an OIE on $\lambda_{a b}$ has to be due to a shift in $n_{s}$ and/or $m_{a b}^{*}$ :

$$
\Delta \lambda_{a b}^{-2}(0) / \lambda_{a b}^{-2}(0)=\Delta n_{s} / n_{s}-\Delta m_{a b}^{*} / m_{a b}^{*} .
$$

Therefore a possible mass dependence of $m_{a b}^{*}$ can be tested by investigating the isotope effect on $\lambda_{a b}$, provided that the contribution of $n_{s}$ to the total isotope shift is known.

The first observation of a possible OIE on the magnetic penetration depth $\lambda(0)$ in polycristalline $\mathrm{YBa}_{2} \mathrm{Cu}_{3} \mathrm{O}_{6.94}$ was reported by Zhao and Morris [39]. In another study Zhao et al. [40, 16] extracted the isotope dependence on $\lambda(0)$ in fine grained samples of $\mathrm{La}_{2-x} \mathrm{Sr}_{x} \mathrm{CuO}_{4}(0.06 \leq x \leq 0.15)$ from the Meissner fraction. Hofer et al. 41] investigated the OIE on $\lambda_{a b}^{-2}(0)$ in tiny single crystals of $\mathrm{La}_{2-x} \mathrm{Sr}_{x} \mathrm{CuO}_{4}$ by means of torque magnetometery. All these experiments showed indeed a pronounced oxygen isotope dependence of the magnetic penetration depth $\lambda$.

The $\mu \mathrm{SR}$ technique is a very powerful method to determine the magnetic penetration depth in superconductors. In the following we will discuss in more detail OIE investigations of $\lambda_{a b}$ in optimally doped and underdoped cuprate HTS by means of bulk $\mu \mathrm{SR}$ and LE $\mu \mathrm{SR}$, which at present is the most powerful and elegant technique to measure the magnetic penetration depth directly. Detailed bulk $\mu \mathrm{SR}$ investigations of polycrystalline samples of HTS have demonstrated that $\lambda$ can be obtained from the muon-spin depolarization rate $\sigma(T) \sim 1 / \lambda^{2}(T)$, which probes the second moment of the magnetic field distribution in the mixed state [42. It was shown [43, 44] that in polycrystalline samples of highly anisotropic systems such as the $\operatorname{HTS}\left(\lambda_{c} / \lambda_{a b}>5\right)$, $\lambda_{\text {eff }}$ (powder awerage) is dominated by the shorter penetration depth $\lambda_{a b}$ due to the supercurrents flowing in the $\mathrm{CuO}_{2}$ planes: $\sigma(T) \propto 1 / \lambda_{a b}^{2}(T)$. In LE $\mu \mathrm{SR}$ experiments spin-polarized low-energy muons are implanted in the thin film sample at a known depth $z$ beneath the surface and precess in the local magnetic field $B(z)$. This feature allows to measure directly the profile $B(z)$ of the magnetic field inside the superconducting film in the Meissner state and to make a model independent determination of $\lambda$ [45. All the 
OIE $\mu$ SR results of $T_{c}$ and $\lambda_{a b}$ discussed in this review are summarized in Table 11. For comparison the low-field magnetization data are also included.

Table 1. Summary of the OIE results.

\begin{tabular}{lllcc}
\hline Compound & Method & $\begin{array}{l}\text { Sample } \\
\text { shape }\end{array}$ & $\begin{array}{c}\Delta T_{c} / T_{c} \\
(\%)\end{array}$ & $\begin{array}{c}\Delta \lambda_{a b}(0) / \lambda_{a b}(0) \\
(\%)\end{array}$ \\
\hline $\mathrm{YBa}_{2} \mathrm{Cu}_{3} \mathrm{O}_{7-\delta}$ & LE $\mu \mathrm{SR}$ & thin film & $-0.22(16)$ & $2.8(7)$ \\
\hline $\mathrm{YBa}_{2} \mathrm{Cu}_{3} \mathrm{O}_{7-\delta}$ & magnetization & fine powder & $-0.26(5)$ & $3.0(1.1)$ \\
& & & $-0.28(5)^{\mathrm{a}}$ & $2.4(1.0)^{\mathrm{a}}$ \\
\hline $\mathrm{YBa}_{2} \mathrm{Cu}_{3} \mathrm{O}_{7-\delta}$ & bulk $\mu \mathrm{SR}$ & powder & $-0.3(1)$ & $2.6(5)$ \\
$\mathrm{Y}_{0.8} \mathrm{Pr}_{0.2} \mathrm{Ba}_{2} \mathrm{Cu}_{3} \mathrm{O}_{7-\delta}$ & & & $-1.3(3)$ & $2.4(7)$ \\
$\mathrm{Y}_{0.7} \mathrm{Pr}_{0.3} \mathrm{Ba}_{2} \mathrm{Cu}_{3} \mathrm{O}_{7-\delta}$ & & & $-2.8(5)$ & $2.5(1.0)$ \\
$\mathrm{Y}_{0.6} \mathrm{Pr}_{0.4} \mathrm{Ba}_{2} \mathrm{Cu}_{3} \mathrm{O}_{7-\delta}$ & & & $-4.6(6)$ & $4.5(1.0)$ \\
$\mathrm{La}_{1.85} \mathrm{Sr}_{0.15} \mathrm{CuO}_{4}$ & & & $-1.0(1)$ & $2.2(6)$ \\
\hline $\mathrm{Y}_{0.6} \mathrm{Pr}_{0.4} \mathrm{Ba}_{2} \mathrm{Cu}_{3} \mathrm{O}_{7-\delta}$ & bulk $\mu \mathrm{SR}$ & site-selective & $0.1(4)^{b}$ & $0.9(5)^{b}$ \\
& & powder & $-3.7(4)^{c}$ & $3.1(5)^{c}$ \\
& & & $-3.3(4)^{d}$ & $3.3(4)^{d}$ \\
\hline
\end{tabular}

${ }^{\text {a }}$ results for the back-exchange ${ }^{16} \mathrm{O} \rightarrow{ }^{18} \mathrm{O}$ and ${ }^{18} \mathrm{O} \rightarrow{ }^{16} \mathrm{O}$ samples

${ }^{\mathrm{b}}$ results for the sample with ${ }^{16} \mathrm{O}$ at plane sites and ${ }^{18} \mathrm{O}$ at apical and chain sites

${ }^{\mathrm{c}}$ results for completely ${ }^{18} \mathrm{O}$ substituted sample

${ }^{\mathrm{d}}$ results for the sample with ${ }^{18} \mathrm{O}$ at plane sites and ${ }^{16} \mathrm{O}$ at apical and chain sites

\subsection{OIE on $\lambda_{a b}$ in underdoped region}

The first measurements of OIE on $\lambda_{a b}(0)$ using bulk $\mu \mathrm{SR}$ were performed by Khasanov et al. 20] in underdoped $\mathrm{Y}_{1-x} \mathrm{Pr}_{x} \mathrm{Ba}_{2} \mathrm{Cu}_{3} \mathrm{O}_{7-\delta}(x=0.3$ and $x=0.4)$. The transversefield $\mu \mathrm{SR}$ measurements were performed on the beam-line $\pi M 3$ at the Paul Scherrer Institute (PSI, Switzerland) using low-momentum muons $(29 \mathrm{MeV} / \mathrm{c})$. The samples were field-cooled from far above $T_{c}$ in a magnetic field of $200 \mathrm{mT}$. The depolarization rate $\sigma$ was extracted from the $\mu \mathrm{SR}$ time spectra using a Gaussian relaxation function $R(t) \propto \exp \left(-\sigma^{2} t^{2} / 2\right)$. Above $T_{c}$ a small temperature-independent depolarization rate $\sigma_{n m}=0.15 \mu s^{-1}$ is seen, arising from the nuclear magnetic moments. Below $T_{c}, \sigma$ increases strongly due to the flux lattice formation. An additional sharp increase of $\sigma(T)$ was observed below $10 \mathrm{~K}$ which is due to antiferromagnetic ordering of $\mathrm{Cu}(2)$ moments 46]. However, zero-field $\mu \mathrm{SR}$ experiments indicate no presence of magnetism above $10 \mathrm{~K}$. Therefore, data points below $10 \mathrm{~K}$ were excluded in the analysis. The 
superconducting contribution $\sigma_{s c}$ was then determined by subtracting $\sigma_{n m}$ measured above $T_{c}$ from $\sigma$.
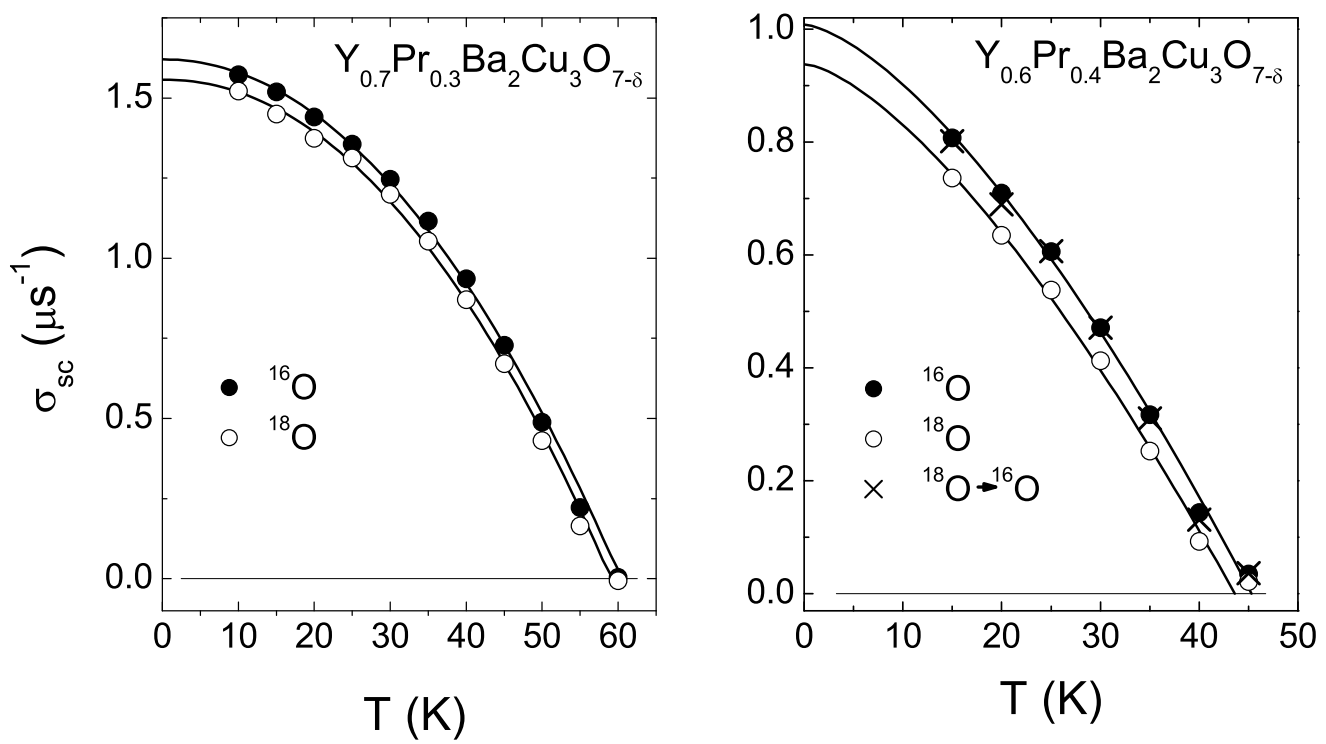

Figure 5. Temperature dependence of the $\mu \mathrm{SR}$ depolarization rate $\sigma_{s c}$ of $\mathrm{Y}_{1-x} \mathrm{Pr}_{x} \mathrm{Ba}_{2} \mathrm{Cu}_{3} \mathrm{O}_{7-\delta}(x=0.3$ and $x=0.4)$ measured in a field $200 \mathrm{mT}(\mathrm{FC})$. The error bars are smaller than the size of the data points. Data points below $10 \mathrm{~K}$ are not shown (see text for an explanation). The solid lines correspond to fits to the power law [see Eq. (7)]. After [20].

In Fig. [5 the temperature dependence of $\sigma_{s c}$ for $\mathrm{Y}_{1-x} \mathrm{Pr}_{x} \mathrm{Ba}_{2} \mathrm{Cu}_{3} \mathrm{O}_{7-\delta}(x=0.3$ and $x=0.4)$ is shown. It is evident that for both concentrations $x$ a remarkable oxygen isotope shift on $T_{c}$ as well as on $\sigma_{s c}(0)$ is observed. The data in Fig. 5 were fitted to the power law 42

$$
\sigma_{s c}(T) / \sigma_{s c}(0)=1-\left(T / T_{c}\right)^{n} .
$$

The values of $\sigma_{s c}(0)$ obtained from the fits were found to be in agreement with previous results [46. In order to prove that the observed OIE on $\lambda_{a b}$ is intrinsic, the ${ }^{18} \mathrm{O}$ sample with $x=0.4$ was back exchanged $\left({ }^{18} \mathrm{O} \rightarrow{ }^{16} \mathrm{O}\right)$. As seen in Fig. 5 the data points of this sample (crosses) coincide with those of the ${ }^{16} \mathrm{O}$ sample. From the measured values of $\sigma_{s c}(0)$ the relative isotope shift of $\lambda_{a b}(0)$ was found to be $\Delta \lambda_{a b}(0) / \lambda_{a b}(0)=2.5(1) \%$ and $4.5(1) \%$ for $x=0.3$ and $x=0.4$ samples, respectively (see Table 1). For the OIE exponent $\beta_{\mathrm{O}}=-d \ln \lambda_{a b}^{-2} / d \ln M_{\mathrm{O}}$ one readily obtains $\beta_{\mathrm{O}}=0.38(12)$ for $x=0.3$ and $\beta_{\mathrm{O}}=0.71(14)$ for $x=0.4$. This means that in underdoped $\mathrm{Y}_{1-x} \operatorname{Pr}_{x} \mathrm{Ba}_{2} \mathrm{Cu}_{3} \mathrm{O}_{7-\delta}$ the OIE on $\lambda_{a b}^{-2}(0)$ as well on $T_{c}$ increases with decreasing of doping. This is in excellent agreement with the magnetic torque results of underdoped $\mathrm{La}_{2-x} \mathrm{Sr}_{x} \mathrm{CuO}_{4}$ single crystals 41. 
Having established the existence of OIE on $\lambda_{a b}$, the following fundamental question arises: Which phonon modes are responsible for this effect? Insight in this respect can be obtained from studying the site-selective OIE (SOIE) on $\lambda_{a b}$. Khasanov et al. 21] performed detailed studies of the SOIE in underdoped $\mathrm{Y}_{0.6} \mathrm{Pr}_{0.4} \mathrm{Ba}_{2} \mathrm{Cu}_{3} \mathrm{O}_{7-\delta}$ polycrystalline samples by bulk $\mu \mathrm{SR}$. In a series of experiments ${ }^{16} \mathrm{O}\left({ }^{18} \mathrm{O}\right)$ oxygen was selectively substituted by ${ }^{18} \mathrm{O}\left({ }^{16} \mathrm{O}\right)$ at apical $(a)$ and chain $(c)$ sites, while ${ }^{16} \mathrm{O}\left({ }^{18} \mathrm{O}\right)$ was kept at plane (p) sites, following the same procedure as described in Refs. 25, 24]. The site-selectivity of the oxygen exchange was checked by Raman spectroscopy [see Fig. [2 (b)]. Figure [6 shows the temperature dependence of the $\mu \mathrm{SR}$ depolarization rate

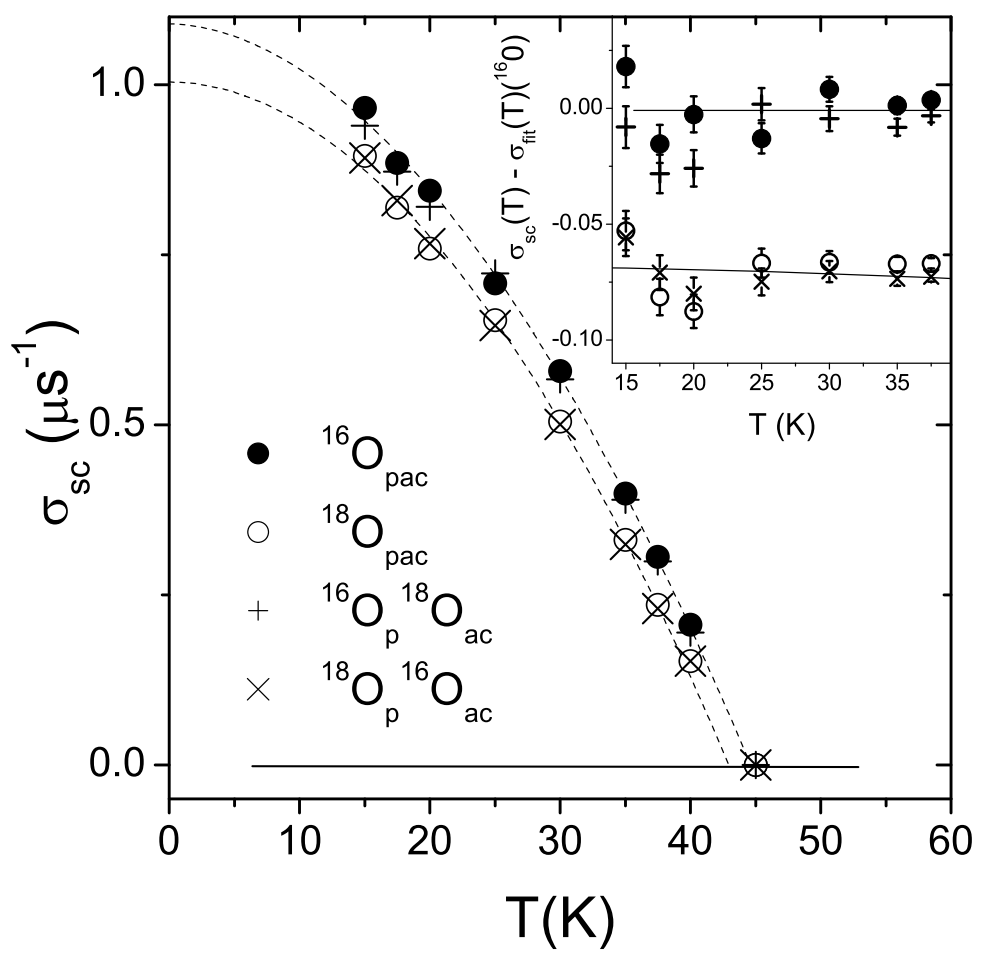

Figure 6. Temperature dependence of the depolarization rate $\sigma_{s c}$ in site-selective $\mathrm{Y}_{0.6} \mathrm{Pr}_{0.4} \mathrm{Ba}_{2} \mathrm{Cu}_{3} \mathrm{O}_{7-\delta}$ samples $(200 \mathrm{mT}, \mathrm{FC})$. Data points below $10 \mathrm{~K}$ are not shown (see text for an explanation). The solid lines correspond to fits to the power law $\sigma_{s c}(T) / \sigma_{s c}(0)=1-\left(T / T_{c}\right)^{n}$ for the ${ }^{16} \mathrm{O}_{\mathrm{pac}}$ and ${ }^{18} \mathrm{O}_{\mathrm{pac}}$ samples. The inset shows data after subtracting the fitted curve for the ${ }^{16} \mathrm{O}_{\text {pac }}$ sample. After [21].

$\sigma_{s c}$ for the $\mathrm{Y}_{0.6} \mathrm{Pr}_{0.4} \mathrm{Ba}_{2} \mathrm{Cu}_{3} \mathrm{O}_{7-\delta}$ site-selective substituted samples. It is evident that a remarkable oxygen isotope shift of $T_{c}$ as well as of $\sigma_{s c}$ is present. More importantly, the data points of the site-selective ${ }^{16} \mathrm{O}_{\mathrm{p}}{ }^{18} \mathrm{O}_{\mathrm{ac}}\left({ }^{18} \mathrm{O}_{\mathrm{p}}{ }^{16} \mathrm{O}_{\mathrm{ac}}\right)$ samples coincide with those of the ${ }^{16} \mathrm{O}_{\text {pac }}\left({ }^{18} \mathrm{O}_{\text {pac }}\right)$ samples. In order to substantiate these results, the power law curve [Eq. (7)] fitting $\sigma_{s c}(T)$ for ${ }^{16} \mathrm{O}_{\mathrm{pac}}$ was subtracted from the experimental data (inset 
in Fig. 6). It can be seen that the experimental points for the two pairs of samples mentioned above coincide within error bar, indicating that the oxygen site within the $\mathrm{CuO}_{2}$ planes mainly contribute to the total OIE on $T_{c}$ and $\lambda_{a b}$.

\subsection{OIE on $\lambda_{a b}$ in optimally doped compounds}

Recently, a substantial OIE on $\lambda_{a b}$ in optimally doped samples of $\mathrm{YBa}_{2} \mathrm{Cu}_{3} \mathrm{O}_{7-\delta}$ $\left[\Delta \lambda_{a b}(0) / \lambda_{a b}(0)=2.6(5) \%, \quad \beta_{\mathrm{O}}=0.41(7)\right]$ (see Fig. (7) and $\mathrm{La}_{1.85} \mathrm{Sr}_{0.15} \mathrm{CuO}_{4}$ $\left[\Delta \lambda_{a b}(0) / \lambda_{a b}(0)=2.2(6) \%, \beta_{\mathrm{O}}=0.35(9)\right]$ was observed by means of bulk $\mu \mathrm{SR}$ [47] (see Table 11). The observation of an OIE on $\lambda_{a b}$ in optimally doped materials is remarkable considering the small OIE on $T_{c}\left[\Delta T_{c} / T_{c}=-0.26(5) \%, \alpha_{\mathrm{O}} \simeq 0.03\right]$. The result is also in accordance with magnetization results obtained for optimally doped $\mathrm{Bi}_{1.6} \mathrm{~Pb}_{0.4} \mathrm{Sr}_{2} \mathrm{Ca}_{2} \mathrm{Cu}_{3} \mathrm{O}_{10+\delta}$ [48].

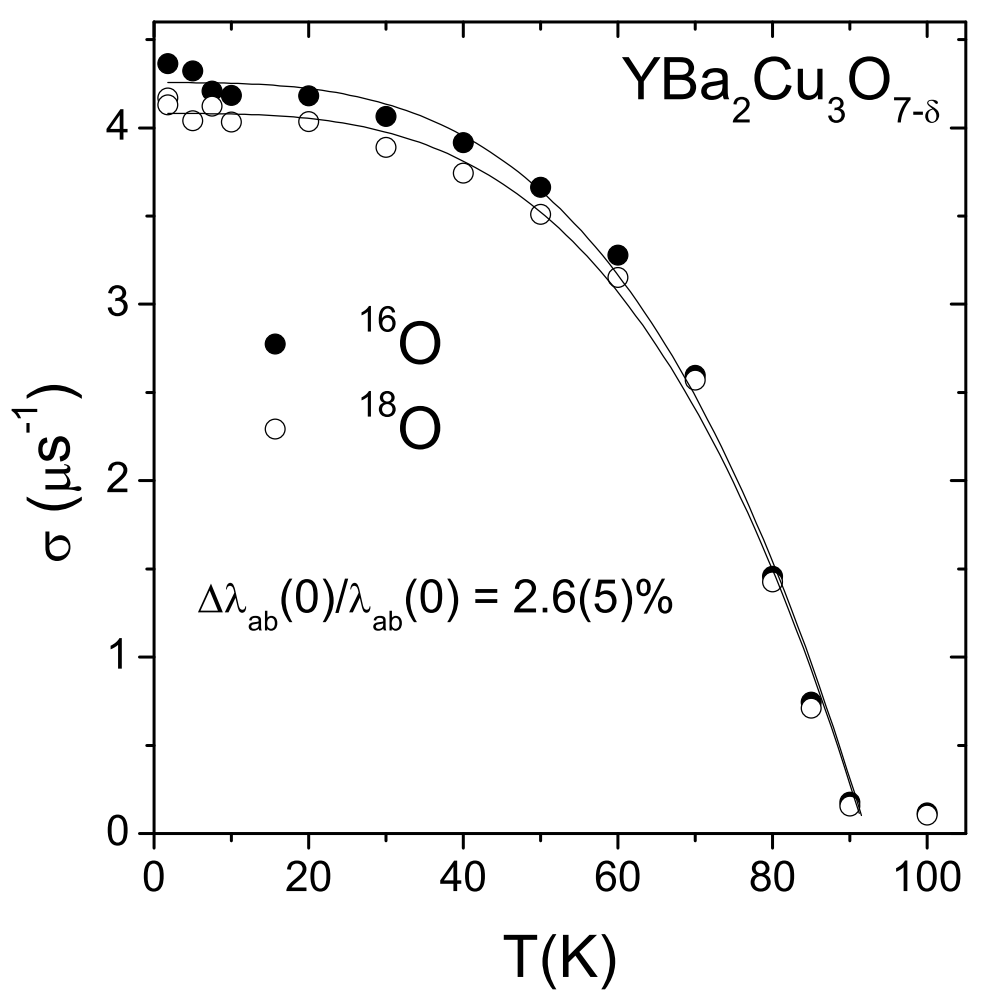

Figure 7. Temperature dependence of the $\mu \mathrm{SR}$ depolarization rate $\sigma$ of oxygen isotope substituted $\mathrm{YBa}_{2} \mathrm{Cu}_{3} \mathrm{O}_{7-\delta}$ measured in a field $200 \mathrm{mT}$ (FC). The error bars are smaller than the size of the data points. The solid lines correspond to fits to the power law [Eq. (7)].

The most direct and model independent way to confirm this result is the determination of $\lambda$ from the functional dependence of magnetic field profile $B(z)$ 
penetrating the surface of a superconductor in the Meissner state. Recently, such a measurement of $\lambda_{a b}$ was performed in a thin film of $\mathrm{YBa}_{2} \mathrm{Cu}_{3} \mathrm{O}_{7-\delta}$ at the Paul Scherrer Institute (PSI, Switzerland) [45, by using the novel low-energy $\mu \mathrm{SR}$ (LE $\mu \mathrm{SR}$ ) technique 49. The principle of the measurement is shown schematically in Fig. 8, Polarized muons of energy in the $\mathrm{keV}$ range are implanted into the film. By tuning their energy these particles can be stopped at different and controllable depths beneath the surface of the superconductor in the Meissner state. The stopping profile of the muons, shown in the bottom part of Fig. 8 (a), depends on the energy and can be reliably simulated [50, 51].
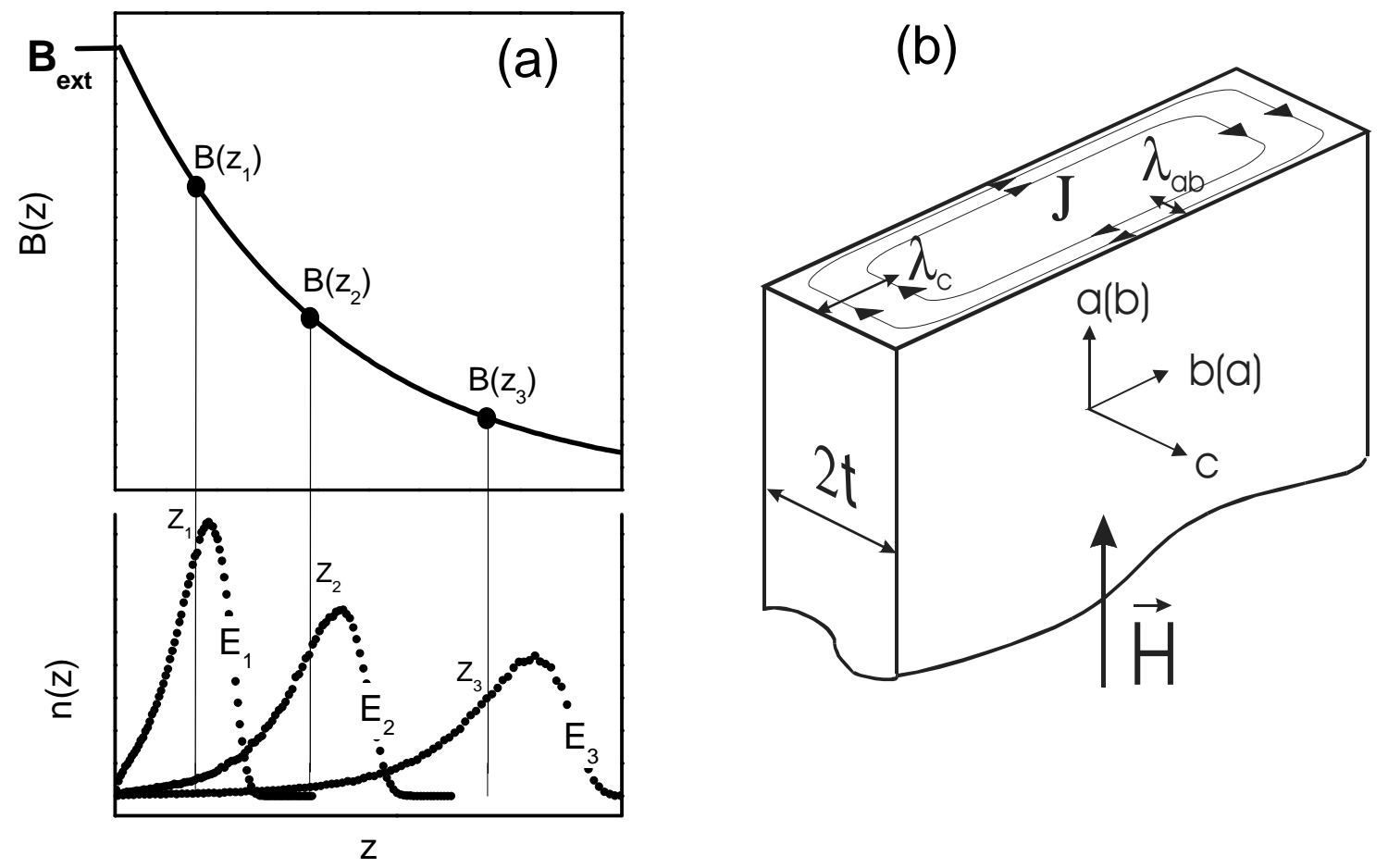

Figure 8. (a) Principle of $\lambda$ determination by low-energy $\mu$ SR. By tuning the energy of muons they are implanted at controllable distances $\left(\bar{z}_{1}, \bar{z}_{2}\right.$, or $\left.\bar{z}_{3}\right)$ from the surface of the superconductor in a Meissner state. The local magnetic field $B(\bar{z})$ is determined from the muon precession frequency.(b) Schematic distribution of the screening current in a thin anisotropic superconducting slab of thickness $2 t$ in a magnetic field applied parallel to the flat surface. The screening current $J$ flows preferably parallel to the $a b$ planes, giving rise to an exponential field decay along the crystal $c$-axis. Due to twinning in the $a b$ planes ( $a$ and $b$ axes are not distinguishable) the so called in-plane magnetic penetration depth $\lambda_{a b}$ is measured.

By means of this technique, measurements of OIE on $\lambda_{a b}$ in optimally doped c-axis oriented $\mathrm{YBa}_{2} \mathrm{Cu}_{3} \mathrm{O}_{7-\delta}$ thin films were recently performed. A weak external magnetic field of $9.2 \mathrm{mT}$ was applied parallel to the sample surface after the sample was cooled in 
zero magnetic field from a temperature above $T_{c}$ to $4 \mathrm{~K}$. In this geometry (the thickness of the sample is negligible in comparison with the width), currents flowing in the $a b$ planes determine the magnetic field profile along the crystal $c$-axis inside the film [see Fig. 8(b)]. Spin-polarized muons were implanted at depths ranging from 20-150 nm beneath the surface of the film by varying the energy of the incident muons from 3 to $30 \mathrm{keV}$. For each implantation energy the average value of the magnetic field $\bar{B}$ and the

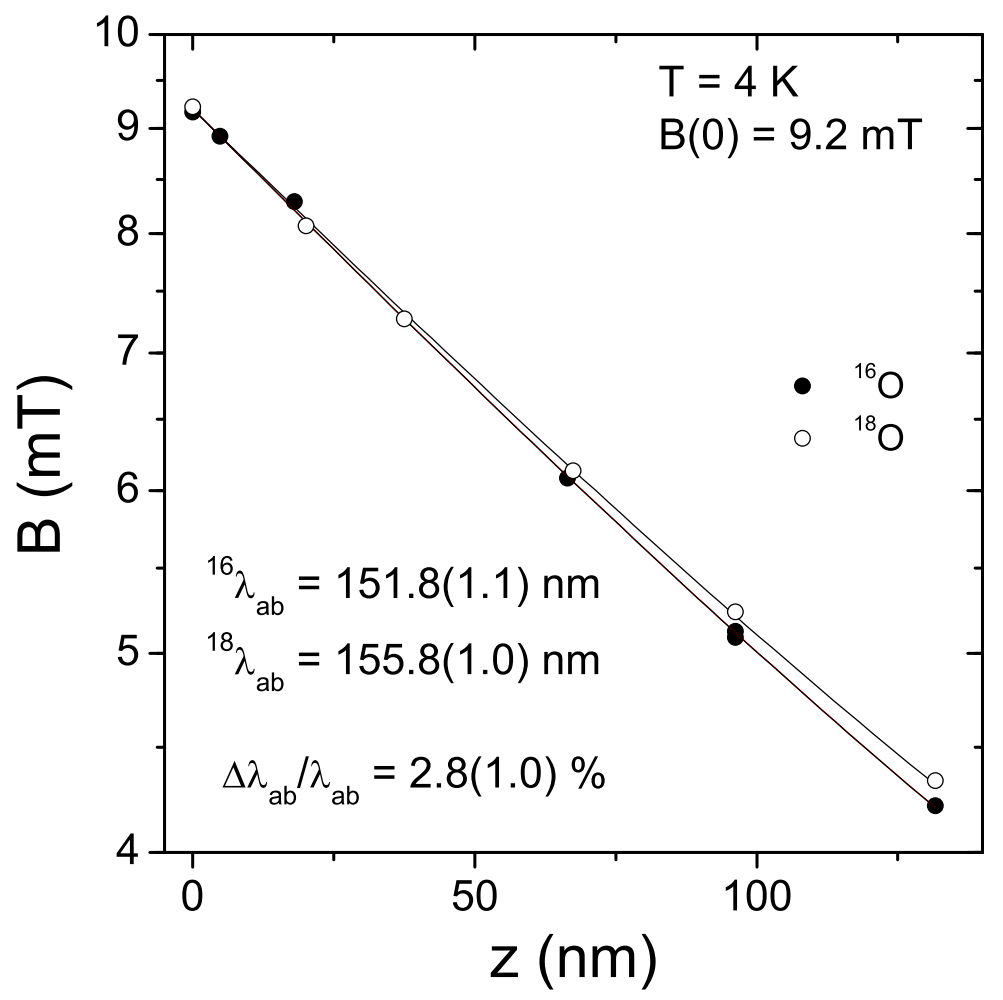

Figure 9. Magnetic field penetration profiles $B(z)$ on a logarithmic scale for a ${ }^{16} \mathrm{O}$ substituted (closed symbols) and a ${ }^{18} \mathrm{O}$ substituted (open symbols) $\mathrm{YBa}_{2} \mathrm{Cu}_{3} \mathrm{O}_{7-\delta}$ film measured in the Meissner state at $4 \mathrm{~K}$ and an external field of $9.2 \mathrm{mT}$, applied parallel to the surface of the film. The data are shown for implantation energies $3,6,10,16$, 22 , and $29 \mathrm{keV}$ starting from the surface of the sample. Solid curves are best fits by Eq. (8). Error bars are smaller than the size of symbols. After [22].

correspondent average value of the stopping distance $\bar{z}$ were extracted. The value of $\bar{B}$ was taken from the fit of the time evolution of the muon-spin polarization spectrum by using the Gaussian relaxation function and $\bar{z}$ was the first moment of the simulated $n(z)$ distribution. Results of this analysis for the ${ }^{16} \mathrm{O}$ and ${ }^{18} \mathrm{O}$ substituted $\mathrm{YBa}_{2} \mathrm{Cu}_{3} \mathrm{O}_{7-\delta}$ films are shown in Fig. 9. The magnetic penetration at the surface of the superconductor is more pronounced for the ${ }^{18} \mathrm{O}$ substituted film showing immediately that ${ }^{18} \lambda_{a b}>{ }^{16} \lambda_{a b}$. 
The solid lines represent a fit to the $\bar{B}$ data by the function:

$$
B(z)=B(0) \frac{\cosh \left[(t-z) / \lambda_{a b}\right]}{\cosh \left(t / \lambda_{a b}\right)} .
$$

This is the form of the classical exponential field decay in the Meissner state $B(z)=$ $B(0) \exp \left(-z / \lambda_{a b}\right)$ (where $B(0)$ is the field at the surface of the superconductor), modified for a film with thickness $2 t$ with flux penetrating from both sides. The value of $z$ was corrected in order to take into account the surface roughness of the films [45, which was taken equal $(8.0(5) \mathrm{nm})$ for both samples originating from the same batch. Fits with Eq. (8) to the extracted ${ }^{16} \bar{B}(\bar{z})$ and ${ }^{18} \bar{B}(\bar{z})$ yield ${ }^{16} \lambda_{a b}(4 \mathrm{~K})=151.8(1.1) \mathrm{nm}$ and ${ }^{18} \lambda_{a b}(4 \mathrm{~K})=155.8(1.0) \mathrm{nm}$. Taking into account a ${ }^{18} \mathrm{O}$ content of $95 \%$, the relative shift is found to be $\Delta \lambda_{a b} / \lambda_{a b}=\left({ }^{18} \lambda_{a b}-{ }^{16} \lambda_{a b}\right) /{ }^{16} \lambda_{a b}=2.8(1.0) \%$ at $4 \mathrm{~K}$. This value is consistent with earlier estimates of the OIE on $\lambda$ for optimally doped $\mathrm{YBa}_{2} \mathrm{Cu}_{3} \mathrm{O}_{7-\delta}$ 39, 31, $\mathrm{La}_{1.85} \mathrm{Sr}_{0.15} \mathrm{CuO}_{4}$ [48] and $\mathrm{Bi}_{1.6} \mathrm{~Pb}_{0.4} \mathrm{Sr}_{2} \mathrm{Ca}_{2} \mathrm{Cu}_{3} \mathrm{O}_{10+\delta}$ [48] extracted indirectly from magnetization measurements.

In order to prove the intrinsic character of the effect, a backexchange experiment was performed on fine powder samples, where the isotope dependence of $\lambda_{a b}(0)$ can be determined from that of the Meissner fraction $f$ 22]. Such type of measurements have been previously performed on the $\mathrm{La}_{2-x} \mathrm{Sr}_{x} \mathrm{CuO}_{4}$ system in a wide range of doping $(0.06 \leq x \leq 0.15)$ [16, 40]. Figure [10] shows the temperature dependence of $\lambda_{a b}^{-2}$ calculated from $f$ for the ${ }^{16} \mathrm{O} /{ }^{18} \mathrm{O}$ substituted $\mathrm{YBa}_{2} \mathrm{Cu}_{3} \mathrm{O}_{7-\delta}$ fine powder samples. The value of $f$ was determined from the FC SQUID magnetization measurements taken at $1 \mathrm{mT}$. The absence of weak links between grains is confirmed by the linear magnetic field dependence of the FC magnetization measured at $5 \mathrm{~K}$ in $0.5 \mathrm{mT}, 1 \mathrm{mT}$, and $1.5 \mathrm{mT}$. $\lambda_{a b}(T)$ can be determined from the measured $\lambda_{\text {eff }}(T)$ using the relation $\lambda_{\text {eff }}=1.31 \lambda_{a b}$ which holds for highly anisotropic superconductors $\left(\lambda_{c} / \lambda_{a b}>5\right)$ [43, 44. The relative shift at $4 \mathrm{~K}$ is found to be $\Delta \lambda_{a b} / \lambda_{a b}=3.0(1.1) \%$, in good agreement with the bulk $\mu \mathrm{SR}$ and LE $\mu \mathrm{SR}$ data (see Figs. [7, 9] and Table 11). The results of the back exchange experiments shown as crosses in Fig. 10 prove the intrinsic character of the effect.

\section{Implications of the OIE on $\lambda_{a b}$ and universal correlations between OIE on $T_{c}$ and $\lambda_{a b}$}

From the present investigations it is evident that there is an OIE on $\lambda_{a b}(0)$ at all doping levels which appears to be generic for cuprate HTS. Here the fundamental questions arise: Is the observation of an OIE on the zero-temperature penetration depth (superfluid density) a direct signature of strong lattice effects? And what is the relevance of this finding for the pairing mechanism? In order to adequately answer these questions more experimental, and in particular more theoretical, work is needed. In the following we only discuss a few points related to these questions.

Under the assumption that we can parameterize experimental data of $\lambda_{a b}(0)$ in terms of Eq. (5) it follows from Eq. (6) that the isotope shift of $\lambda_{a b}$ is due to an isotope shift of $n_{s}$ and/or $m_{a b}^{*}$. It was demonstrated by a number of independent experiments 


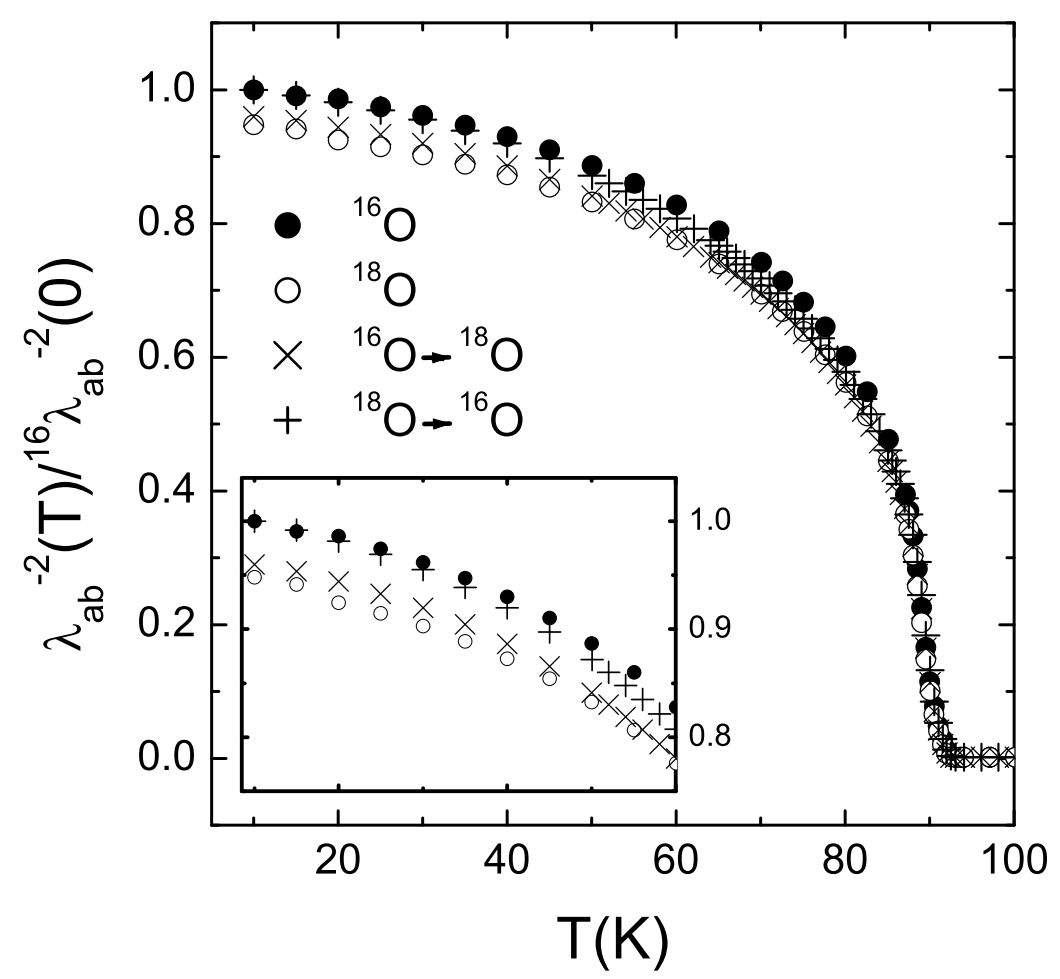

Figure 10. Temperature dependence of $\lambda_{a b}^{-2}$ normalized by ${ }^{16} \lambda_{a b}^{-2}(0)$ for ${ }^{16} \mathrm{O}$ and ${ }^{18} \mathrm{O}$ substituted $\mathrm{YBa}_{2} \mathrm{Cu}_{3} \mathrm{O}_{7-\delta}$ fine powder samples as obtained from low-field SQUID magnetization measurements. The inset shows the low-temperature region between $10 \mathrm{~K}$ and $60 \mathrm{~K}$. The reproducibility of the oxygen exchange procedure was checked by the backexchange (crosses). After [22].

that for $\mathrm{La}_{2-x} \mathrm{Sr}_{x} \mathrm{CuO}_{4}$ [16, 18, 40, 41] and $\mathrm{Y}_{1-x} \mathrm{Pr}_{x} \mathrm{Ba}_{2} \mathrm{Cu}_{3} \mathrm{O}_{7-\delta}$ [20] the change of $n_{s}$ during the oxygen exchange procedure is negligibly small. Recently, Khasanov et al. [22] provided further evidence of this scenario from measurements of the nuclear quadrupole resonance (NQR) frequency of the plane and the chain ${ }^{63} \mathrm{Cu}$ in ${ }^{16} \mathrm{O}$ and ${ }^{18} \mathrm{O}$ substituted optimally doped $\mathrm{YBa}_{2} \mathrm{Cu}_{3} \mathrm{O}_{7-\delta}$ powder samples. It was found that the change of the hole number per unit cell caused by the isotope substitution is less than $\simeq 10^{-3}$. Since in optimally doped $\mathrm{YBa}_{2} \mathrm{Cu}_{3} \mathrm{O}_{7-\delta}$ there is approximately one doped hole per unit cell, the relative change of the hole density $\Delta n / n$ at the oxygen exchange must be less than $10^{-3}$. The absence of an observable OIE on $n_{s}$ implies that the change of $\lambda_{a b}(0)$ is mainly due to a change on $m_{a b}^{*}$. From Eqs. (5) and (6) it follows that

$$
\Delta m_{a b}^{*} / m_{a b}^{*} \simeq-\Delta \lambda_{a b}^{-2}(0) / \lambda_{a b}^{-2}(0)=2 \Delta \lambda_{a b}(0) / \lambda_{a b}(0) .
$$

This implies that in HTS $m_{a b}^{*}$ depends on the oxygen mass $M_{\mathrm{O}}$ with $\Delta m_{a b}^{*} / m_{a b}^{*} \simeq$ $5-10 \%$, depending on doping level (see also Table 1). Note that such an isotope effect on $m_{a b}^{*}$ is not expected for a conventional weak-coupling phonon-mediated BCS superconductor. In fact in HTS the charge carriers are very likely coupled to optical 
phonons, as indicated by measurements of the static and high-frequency dielectric constants [52, neutron scattering [7, 8, photoemission [9, 10, 53] and tunnelling [54] experiments. Strong interaction between the charge carriers and the lattice ions leads to a break down of the adiabatic Migdal approximation [13, and consequently the effective supercarrier mass $m^{*}$ depends on the mass of the lattice atoms. To our knowledge there are just a few theoretical models which predict an OIE on the effective carrier mass $m^{*}$ (see e.g. Refs. [13, 55, 56, 57]).

Khasanov et al. [20] reported an empirical linear relation between the OIE exponents $\alpha_{\mathrm{O}}=-d \ln T_{c} / d \ln M_{\mathrm{O}}$ and $\beta_{\mathrm{O}}=-d \ln \lambda_{a b}^{-2}(0) / d \ln M_{\mathrm{O}}$ for underdoped $\mathrm{La}_{2-x} \mathrm{Sr}_{x} \mathrm{CuO}_{4}$ and $\mathrm{Y}_{1-x} \mathrm{Pr}_{x} \mathrm{Ba}_{2} \mathrm{Cu}_{3} \mathrm{O}_{7-\delta}$. In Fig. 11 we plot $\Delta \lambda_{a b}(0) / \lambda_{a b}(0) \propto \beta_{\mathrm{O}}$ versus $-\Delta T_{c} / T_{c} \propto \alpha_{\mathrm{O}}$ for $\mathrm{Y}_{1-x} \mathrm{Pr}_{x} \mathrm{Ba}_{2} \mathrm{Cu}_{3} \mathrm{O}_{7-\delta}$ and $\mathrm{La}_{2-x} \mathrm{Sr}_{x} \mathrm{CuO}_{4}$ for the data listed in Table 1 It is evident from Fig. 11 that there is correlation between the OIE of $T_{c}$ and $\lambda_{a b}(0)$ which appears to be universal for cuprate HTS. Different experimental techniques (SQUID magnetization, magnetic torque, bulk $\mu \mathrm{SR}, \mathrm{LE} \mu \mathrm{SR}$ ) and different types of samples (single crystals, powders, thin films) yield within error bar consisting results, indicating that the observed isotope effects are intrinsic and not artifacts of the particular experimental method or sample used. As indicated by the dashed line, at low doping level $\Delta \lambda_{a b}(0) / \lambda_{a b}(0) \simeq\left|\Delta T_{c} / T_{c}\right|$, whereas close to optimal doping $\Delta \lambda_{a b}(0) / \lambda_{a b}(0)$ is almost constant and considerably larger than $\left|\Delta T_{c} / T_{c}\right|$ $\left(\Delta \lambda_{a b}(0) / \lambda_{a b}(0) \approx 10\left|\Delta T_{c} / T_{c}\right|\right)$. This finding is consistent with the empirical "Uemura relation " [58, 59] and with the "parabolic ansatz" proposed in [30] in a differential way for doped cuprate HTS.

It was shown by Schneider and Keller [60, 61] that this empirical relation naturally follows from the doping driven 3D-2D crossover and the 2D quantum superconductor to insulator (2D-QSI) transition in the highly underdoped limit. Close to the 2D-QSI transition the following relation holds [61]:

$$
\Delta \lambda_{a b}(0) / \lambda_{a b}(0)=(1 / 2)\left[\Delta d_{s} / d_{s}-\Delta T_{c} / T_{c}\right],
$$

where $\Delta d_{s} / d_{s}$ is the oxygen-isotope shift of the thickness of the superconducting sheets $d_{s}$ of the sample. The solid line in Fig. [11]represents a best fit of Eq. (10) to the data with $\Delta d_{s} / d_{s}=3.3(4) \%$. Since the lattice parameters are not modified by oxygen substitution 62, 63, the observation of an isotope shift of $d_{s}$ implies local lattice distortions involving oxygen which are coupled to the superfluid 61.

As shown in Fig. 12, a similar type of correlation between the OIE on $\lambda_{a b}(0)$ and OIE on $T_{c}$ is also observed for the site-selective substituted $\mathrm{Y}_{0.6} \mathrm{Pr}_{0.4} \mathrm{Ba}_{2} \mathrm{Cu}_{3} \mathrm{O}_{7-\delta}$ samples [21]. It is evident that the oxygen site within the $\mathrm{CuO}_{2}$ planes mainly contributes to the total OIE on $T_{c}$ and $\lambda_{a b}(0)$. Since for fixed $\operatorname{Pr}$ concentration the lattice parameters remain essentially unaffected by the isotope substitution [62, 63], these results unambiguously demonstrate the existence of a coupling of the charge carriers to phonon modes involving movements of the oxygen atoms in the $\mathrm{CuO}_{2}$ plane, while suggesting that modes involving apical and chain oxygen are less strongly coupled to the carriers. 


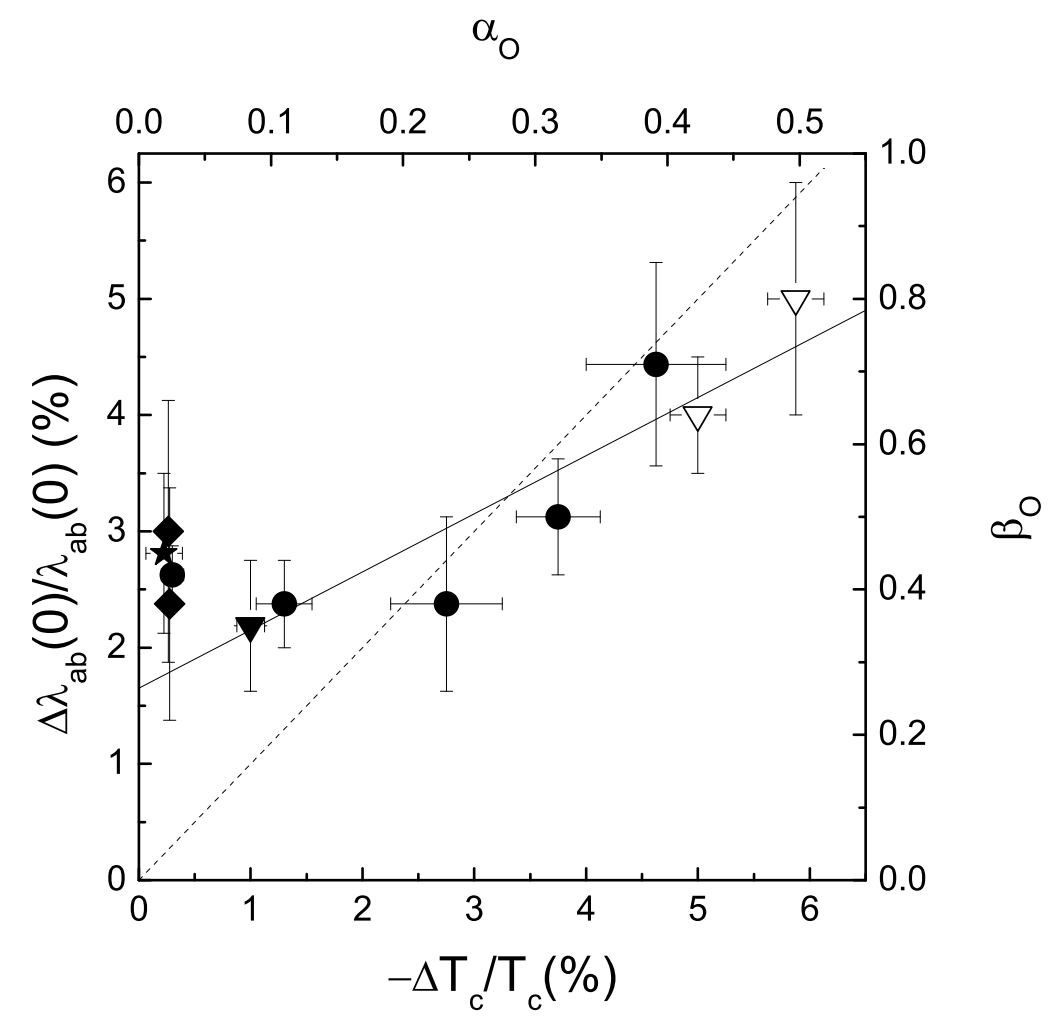

Figure 11. Plot of the OIE shift $\Delta \lambda_{a b}(0) / \lambda_{a b}(0)$ (OIE exponent $\beta_{\mathrm{O}}$ ) versus the OIE shift $-\Delta T_{c} / T_{c}$ (OIE exponent $\alpha_{\mathrm{O}}$ ) for $\mathrm{Y}_{1-x} \mathrm{Pr}_{x} \mathrm{Ba}_{2} \mathrm{Cu}_{3} \mathrm{O}_{7-\delta}$ and $\mathrm{La}_{2-x} \mathrm{Sr}_{x} \mathrm{CuO}_{4}$. Closed circles and triangles are bulk $\mu$ SR data for $\mathrm{Y}_{1-x} \mathrm{Pr}_{x} \mathrm{Ba}_{2} \mathrm{Cu}_{3} \mathrm{O}_{7-\delta}$ and $\mathrm{La}_{1.85} \mathrm{Sr}_{0.15} \mathrm{CuO}_{4}$ (Table 1). Diamonds and stars are $\mathrm{LE} \mu \mathrm{SR}$ and Meissner fraction data for optimally doped $\mathrm{YBa}_{2} \mathrm{Cu}_{3} \mathrm{O}_{7-\delta}$ (Table 1). Open triangles are torque magnetization data for $\mathrm{La}_{2-x} \mathrm{Sr}_{x} \mathrm{CuO}_{4}$ from [4]. The dashed line corresponds to $\Delta \lambda_{a b}(0) / \lambda_{a b}(0)=\left|\Delta T_{c} / T_{c}\right|$. The solid line indicates the flow to 2D-OSI criticality and provides with Eq. (10) an estimate for the oxygen isotope effect on $d_{s}$, namely $\Delta d_{s} / d_{s}=3.3(4) \%$.

\section{Conclusions}

In conclusion, the unconventional isotope effects presented here clearly demonstrate that lattice effects play an important role in the physics of cuprates. The fact that a substantial OIE on $\lambda_{a b}$ is observed, even in optimally doped cuprates, strongly suggests that cuprate HTS are non-adiabatic superconductors. This is in contrast to the novel high-temperature superconductor $\mathrm{MgB}_{2}$ for which within experimental error no Boron $\left({ }^{10} \mathrm{~B} /{ }^{11} \mathrm{~B}\right)$ isotope effect on the magnetic penetration depth was detected 64]. The site-selective OIE studies of $T_{c}$ and $\lambda_{a b}$ indicate that the phonon modes involving the movements of oxygen within the superconducting $\mathrm{CuO}_{2}$ planes are essential for the occurrence of superconductivity in cuprate HTS. This is in agreement with 


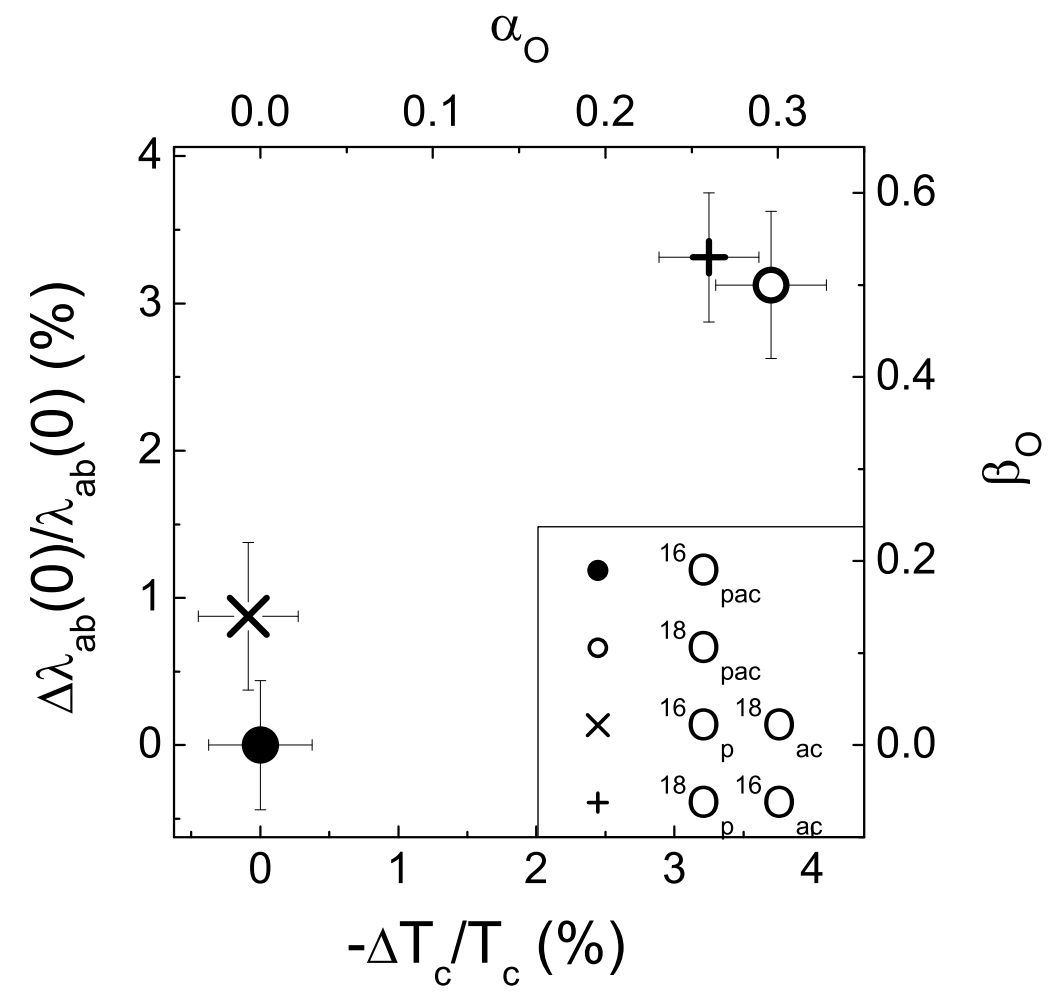

Figure 12. Plot of the OIE shift of the in-plane penetration depth $\Delta \lambda_{a b}(0) / \lambda_{a b}(0)$ (OIE exponent $\beta_{\mathrm{O}}$ ) versus the OIE shift of the transition temperature $-\Delta T_{c} / T_{c}$ (OIE exponent $\alpha_{\mathrm{O}}$ ) for site-selective substituted $\mathrm{Y}_{0.6} \mathrm{Pr}_{0.4} \mathrm{Ba}_{2} \mathrm{Cu}_{3} \mathrm{O}_{7-\delta}$ samples. The error bars of the "trivial" ${ }^{16} \mathrm{O}_{\text {pac }}$ point (zero isotope shift by definition) indicate the intrinsic uncertainty in the determination of $T_{c}$ and $\lambda_{a b}$. After [21.

recent inelastic neutron scattering [7, 8, and photoemission 9, 10, studies, indicating a strong interaction between charge carriers and $\mathrm{Cu}-\mathrm{O}$ bond-stretching-type of phonons. The generic relation between the OIE on $T_{c}$ and $\lambda_{a b}$ demonstrates that, in contrast to conventional phonon-mediated superconductors, $\lambda_{a b}$ (superfluid density) is a key parameter for understanding the role of phonons in cuprates, in particular local lattice distortions involving planar oxygen. The present results rise serious doubts that models, neglecting lattice degrees of freedom, as proposed for instance in [65, are potential candidates to explain superconductivity in HTS.

This work was partly performed at the Swiss Muon Source $(\mathrm{S} \mu \mathrm{S})$, Paul Scherrer Institute (PSI, Switzerland). The authors are grateful to A. Amato U. Zimmermann, and D. Herlach for help during the $\mu \mathrm{SR}$ measurements, D. Di Castro, N. Garifianov, D.G. Eshchenko, H. Luetkens, T. Prokscha, and A. Suter for helpful discussions and participating in some $\mu \mathrm{SR}$ experiments, A. Bussmann-Holder, E. Liarokapis, 
D. Lampakis, M. Mali, K.A. Müller, J. Roos, T. Schneider, Z.-X. Shen, A. Tatsi, and G.M. Zhao for their collaborations and discussions. This work was supported by the Swiss National Science Foundation and by the NCCR program Materials with Novel Electronic Properties (MaNEP) sponsored by the Swiss National Science Foundation.

\section{References}

[1] Bednorz J G and Müller K A 1986 Z. Phys. B 64189

[2] Batlogg B, Cava R J, Jayaraman A, van Dover R B, Kourouklis G A, Sunshine S, Murphy D W, Rupp L W, Chen H S, White A, Short K T, Mujsce A M, and Rietman E A 1986 Phys. Rev. Lett. 582333

[3] Batlogg B, Kourouklis G, Weber W, Cava R J, Jayaraman A, White A E, Short K T, Rupp L W, and Rietman E A 1887 Phys. Rev. Lett. 59912

[4] Bourne L C, Crommie M F, Zettl A, Hans-Conrad zur Loye, Keller S W, Leary K L, Stacy Angelica M Chang K J, Cohen Marvin L, and Morris Donald E 1987 Phys. Rev. Lett. 58 2337

[5] Carbotte J P, 1990 Rev. Mod. Phys. 621027

[6] Nagamatsu J, Nakagawa N, Muranaka T, Zenitani Y, and Akimitsu J 2001 Nature (London) 410 63

[7] McQueeney R J, Sarrao J L, Pagliuso P G, Stephens P W, and Osborn R 1999 Phys. Rev. Lett. 82 628; 200187 077001; d'Astuto M, Mang P K, Giura P, Shukla A, Ghigna P, Mirone A, Braden M, Greven M, Krisch M, and Sette F 2002 ibid. 88167002

[8] Chung J-H, Egami T, McQueeney R J, Yethiraj M, Arai M, Yokoo T, Petrov Y, Mook H A, Endoh Y, Tajima S, Frost C, and Dogan F 2003 Phys. Rev. B 67014517

[9] Bogdanov P V, Lanzara A, Kellar S A, Zhou X J, Lu E D, Zheng W J, Gu G, Shimoyama JI, Kishio K, Ikeda H, Yoshizaki R, Hussain Z, and Shen Z- 2000 Phys. Rev. Lett. 85 2581; Bogdanov P V, Lanzara A, Zhou X J, Yang W L, Eisaki H, Hussain Z, and Shen Z-X 2002 ibid. 89167002

[10] Lanzara A, Bogdanov P V, Zhou X J, Kellar S A, Feng D L, Lu E D, Yoshida T, Eisaki H, Fujimori A, Kishio K, Shimoyama J-I, Noda T, Uchida S, Hussain Z, and Shen Z-X 2001 Nature (London) 412510

[11] Bussmann-Holder A, Müller K A, Micnas R, Büttner H, Simon A, Bishop A R, and Egami T 2001 J. Phys.: Condens. Matter 13 L169; Mihailovic D and Kabanov V V 2001 Phys. Rev. B 63 054505; Shen Z-X et al. 2002 Phil. Mag. B 82 1349; Tachiki M, Machida M, and Egami T 2003 Phys. Rev. B 67174506

[12] Eschrig M and Norman M R 2003 Phys. Rev. B 67 144503; Schachinger E, Tu J J, and Carbotte J P 2003 ibid. $\mathbf{6 7} 214508$

[13] Alexandrov A S and Mott N F 1994 Int. J. Mod. Phys. 82075

[14] Müller K A 1900 Z. Phys. B. 80193

[15] Zech D, Conder K, Keller H, Kaldis E, Liarokapis E, Poulakis N, and Müller K A, in: Anharmonic Properties of High-T $T_{c}$ Cuprates, ed. D. Mihailović, G. Ruani, E. Kaldis, and K.A. Müller (World Scientific, Singapore, 1995), pp. 18-29.

[16] Zhao G M, Conder K, Keller H, and Müller K A 1998 J. Phys.: Condens. Matter 109055

[17] Müller K A 2000 Physica C 341-348 11

[18] Zhao G M, Keller H, and Conder K 2001 J. Phys.: Cond. Matter 13 R569

[19] Keller H 2003 Physica B $\mathbf{3 2 6} 283$

[20] Khasanov R, Shengelaya A, Conder K, Morenzoni E, Savić I M, and Keller H 2003 J. Phys.: Condens Matter 15 L17

[21] Khasanov R, Shengelaya A, Morenzoni E, Angst M, Conder K, Savić I M, Lampakis D, Liarokapis E, Tatsi A, and Keller H 2003 Phys. Rev. B 68220506 
[22] Khasanov R, Eshchenko D G, Luetkens H, Morenzoni E, Prokscha T, Suter A, Garifianov N, Mali M, Roos J, Conder K, and Keller H 2004 Phys. Rev. Lett. 92057602

[23] Morenzoni E, Prokscha Th, Hofer A, Matthias B, Meyberg M, Wutzke Th, Glckler H, Birke M, Litterst J, Niedermayer Ch, Schatz G 1997 J. Appl. Phys. 813340

[24] Conder K 2001 Mater. Sci. Eng. R32 41

[25] Zech D, Keller H, Conder K, Kaldis E, Liarokapis E, Poulakis N, and Müller K A 1994 Nature (London) 371681

[26] Ramirez A P, Kortan A R, Rosseinsky M J, Duclos S J, Mujsce A M, Haddon R C, Murphy D W, Makhija A V, Zahurak S M, and Lyons K B 1992 Phys. Rev. Lett. 681058

[27] Budko S L, Lapertot G, Petrovic C, Cunningham C E, Anderson N, and Canfield P C 2001 Phys. Rev. Lett. 86 1877; Hinks D G, Claus H, and Jorgensen J D 2001 Nature (London) 411 457

[28] Franck J P, Jung J, Mohamed M A-K, Gygax S, and Sproule G I 1991 Phys. Rev. B 445318

[29] Franck J P, in: Physical Properties of High Temperature Superconductors IV, edited by D. M. Ginsberg (World Scientific, Singapore, 1994), pp. 189-293

[30] Schneider T and Keller H 1992 Phys. Rev. Lett. 693374

[31] Zhao G M and Morris D E 1996 Phys. Rev. B 5114982

[32] Bornermann H J and Morris D E 1991 Phys. rev. B 445532

[33] Babushkina N, Inyushkin A, Ozhogin V, Taldenkov A, Kobrin I, Vorob'eva T, Molchanova L, Damyanets L, Uvarova T, and Kuzakov A 1991 Physica C 185-189 901

[34] Franck J P, Harker S, and Brewer J 1993 Phys. Rev. Lett. 71283

[35] Zhao G M, Kirtikar V, Singh K K, Sinha A P B, Morris Donald E, and Inyushkin A V 1996 Phys. Rev. B 5414956

[36] Morris D E, Sinha A P B, Kirtikar V, and Inyushkin A V 1998 Physica C 298203

[37] Williams G V M, Pringle D J, and Tallon J L 2000 Phys. Rev. B 61 R9257

[38] Chandrasekhar B S and Einzel D 1993 Ann. Physik 2535

[39] Zhao G M and Morris D E 1995 Phys. Rev. B 5116487

[40] Zhao G. M, Hunt M B, Keller H, and Müller K A 1997 Nature (London) 385236

[41] Hofer J, Conder K, Sasagawa T, Zhao G M, Willemin M, Keller H, and Kishio K 2000 Phys. Rev. Lett. 844192

[42] Zimmermann P, Keller H, Lee S L, Savic I M, Warden M, Zech D, Cubitt R, Forgan E M, Kaldis E, Karpinski J, and Krüger C 1995 Phys. Rev. B 52541

[43] Barford W and Gunn J M F 1988 Physica C 156515

[44] Fesenko V I, Gorbunov V N, and Smilga V P 1991 Physica C 176551

[45] Jackson T J, Riseman T M, Forgan E M, Glückler H, Prokscha T, Morenzoni E, Pleines M, Niedermayer Ch, Schatz G, Luetkens H, and Litterst J 2000 Phys. Rev. Lett. 844958

[46] Seaman C L, Neumeier J J, Maple M B, Le L P, Luke G M, Sternlieb B J, Uemura Y J, Brewer J H, Kadono R, Kiefl R F, Krietzman S R, and Riseman T M 1990 Phys. Rev. B 426801

[47] Khasanov R et al. unpublished.

[48] Zhao G M, Kirtikar Vidula, and Morris Donald E 2001 Phys. Rev. B 63220506

[49] Morenzoni E, Kottmann F, Maden D, Matthias B, Meyberg M, Prokscha Th, Wutzke Th, and Zimmermann U 1994 Phys. Rev. Lett. 722793

[50] Morenzoni E, Glückler H, Prokscha T, Khasanov R, Luetkens H, Birke M, Forgan E M, and Niedermayer Ch 2002 Nucl. Instr. Meth. B 192254

[51] Morenzoni E, Prokscha T, Suter A, Luetkens H, and Khasanov R (in this issue).

[52] Alexandrov A S and Bratkovsky A M 2000 Phys. Rev. Lett. 842043

[53] Shen Z-X, Lanzara A, Ishihara S, Nagaosa N, cond-mat/0108381

[54] Ponomarev Ya G, Tsokur E B, Sudakova M V, Tchesnokov S N, Shabalin M E, Lorenz M A, Hein M A, Müller G, Piel H, and Aminov B A 1999 Solid State Commun. 111513

[55] Scalapino D J, Scalettar R T, and Bickers N E, in Proceedings of the International Conference on Novel Mechanisms of Superconductivity, New York, 1987, edited by S.E Wolf and V.Z. Kresin 
(Plenum, New York, 1987), p. 475

[56] Grimaldi C, Cappelluti E, and Pietronero L 1998 Europhys. Lett. 42667

[57] Bussmann-Holder A and Micnas R 2002 J. Supercond. 15321

[58] Uemura Y J, Luke G M, Sternlieb B J, Brewer J H, Carolan J F, Hardy W N, Kadono R, Kempton J R, Kiefl R F, Kreitzman S R, Mulhern P, Riseman T M, Williams D Ll, Yang B X, Uchida S, Takagi H, Gopalakrishnan J, Sleight A W, Subramanian A M, Chien C L, Cieplak M Z, Gang Xiao, Lee V Y, Statt B W, Stronach C E, Kossler W J, and Yu X H 1989 Phys. Rev. Lett. 622317

[59] Uemura Y J, Le L P, Luke G M, Sternlieb B J, Wu W D, Brewer J H, Riseman T M, Seaman C L, Maple M B, Ishikawa M, Hinks D G, Jorgensen J D, Saito G, and Yamochi H 1991 Phys. Rev. Lett. 662665

[60] Schneider T and Keller H 2001 Phys. Rev. Lett. 864899

[61] Keller H and Schneider T cond-mat/0401505

[62] Conder K, Zech D, Krüger Ch, Kaldis E, Keller H, Hewat A W, and Jilek E, in Phase Separation in Cuprate Superconductors, edited by E. Sigmund and K. A. Müller (Springer, Berlin 1994) p. 210

[63] Raffa F, Ohno T, Mali M, Roos J, Brinkmann D, Conder K, and Eremin M 1998 Phys. Rev. Lett. 815912

[64] Di Castro D, Angst M, Eshchenko D G, Khasanov R, Roos J, Savić I M, Shengelaya A, Bud'ko S L, Canfield P C, Conder K, Karpinski J, Kazakov S M, Ribeiro R A, and Keller H, cond-mat/0307330

[65] Anderson P W, Lee P A, Randeria M, Rice T M, Trivedi N, and Zhang F C, cond-mat/0311467 